\title{
Targeted walking in incomplete spinal cord injury: Role of corticospinal control
}

Meyer, Christian ; Filli, Linard ; Stalder, Stephanie Anja ; Awai Easthope, Christopher ; Killeen, Tim ; von Tscharner, Vinzenz ; Curt, Armin ; Zörner, Björn ; Bolliger, Marc

\begin{abstract}
Locomotor recovery after incomplete spinal cord injury (iSCI) is influenced by spinal and supraspinal networks. Conventional clinical gait analysis fails to differentiate between these components. There is evidence that corticospinal control is enhanced during targeted walking, where each foot must be continuously placed on visual targets in randomized order. This study investigates the potential of targeted walking in the functional assessment of corticospinal integrity. Twenty-one controls and 16 individuals with chronic iSCI performed normal and targeted walking on a treadmill while electromyograms (EMGs) and kinematics were recorded. Precision (\% of accurate foot placements) in targeted walking was significantly lower in individuals with iSCI $(82.9 \pm 14.7 \%$, controls: $94.9 \pm 4.0 \%)$. While the overall kinematic pattern was comparable between walking conditions, controls showed significantly higher semitendinosus activity before heel-strike during targeted walking. This was accompanied by a shift of relative EMG intensity from $90-120 \mathrm{~Hz}$ to lower frequencies of $20-60 \mathrm{~Hz}$, previously associated with corticospinal control of muscle activity. Targeted walking in iSCI individuals evoked smaller EMG changes, suggesting that the switch to more corticospinal control is impaired. Accordingly, mildly impaired iSCI individuals revealed higher adaptations to the targeted walking task than more-impaired individuals. Recording of EMGs during targeted walking holds potential as a research tool to reveal further insights into the neuromuscular control of locomotion. It also complements findings of preclinical studies and is a promising novel surrogate marker of integrity of corticospinal control in individuals with iSCI and other neurological impairments. Future studies should investigate its potential for diagnosis or tracking recovery during rehabilitation.
\end{abstract}

DOI: https://doi.org/10.1089/neu.2020.7030

Posted at the Zurich Open Repository and Archive, University of Zurich

ZORA URL: https://doi.org/10.5167/uzh-188968

Journal Article

Accepted Version

Originally published at:

Meyer, Christian; Filli, Linard; Stalder, Stephanie Anja; Awai Easthope, Christopher; Killeen, Tim; von Tscharner, Vinzenz; Curt, Armin; Zörner, Björn; Bolliger, Marc (2020). Targeted walking in incomplete spinal cord injury: Role of corticospinal control. Journal of Neurotrauma, 37(21):2302-2314.

DOI: https://doi.org/10.1089/neu.2020.7030 


\section{Targeted walking in incomplete spinal cord injury: Role of corticospinal control}

\section{List of authors:}

Christian Meyer, MSc

Spinal Cord Injury Center, Balgrist University Hospital,Forchstrasse 340, 8008 Zurich, Switzerland, christian.meyer@balgrist.ch, +41 445107217

Linard Filli, PhD

1. Spinal Cord Injury Center, Balgrist University Hospital,Forchstrasse 340, 8008 Zurich, Switzerland, linard.filli@balgrist.ch, +41 445107212

2. Department of Neurology, University Hospital Zurich, Frauenklinikstrasse 26, 8092 Zurich, Switzerland

Stephanie A. Stalder, MSc

Spinal Cord Injury Center, Balgrist University Hospital,Forchstrasse 340, 8008 Zurich, Switzerland, stephanie.stalder@balgrist.ch, +41 443865657

Christopher Awai Easthope, PhD

Spinal Cord Injury Center, Balgrist University Hospital,Forchstrasse 340, 8008 Zurich, Switzerland, chris.awai@cereneo.foundation, +41 413996744

Tim Killeen, MD, PhD

Spinal Cord Injury Center, Balgrist University Hospital,Forchstrasse 340, 8008 Zurich, Switzerland, tim.killeen@doctors.org.uk, +41 445107201

Vinzenz von Tscharner, PhD

Faculty of Kinesiology, University of Calgary, 2500 University Drive N.W., Calgary, Alberta, Canada T2N 14N, tvvon@ucalgary.ca, +1 (403) 220-3436 
Armin Curt, MD

Spinal Cord Injury Center, Balgrist University Hospital,Forchstrasse 340, 8008 Zurich, Switzerland, armin.curt@balgrist.ch, +41 443861111

Björn Zörner, MD, PhD ${ }^{1 *}$

Spinal Cord Injury Center, Balgrist University Hospital,Forchstrasse 340, 8008 Zurich, Switzerland, bjoern.zoerner@balgrist.ch, +41 443865659

Marc Bolliger, $\mathrm{PhD}^{1^{*}}$ (Corresponding author)

Spinal Cord Injury Center, Balgrist University Hospital,Forchstrasse 340, 8008 Zurich, Switzerland, marc.bolliger@balgrist.ch, +41 445107201

* These authors jointly supervised this work and contributed equally to this manuscript.

Word count abstract: 250

Word count manuscript: 6080

Number of figures/tables: 6 (and 3 Supplementary)

Running title: Role of corticospinal control in targeted walking 
Locomotor recovery after incomplete spinal cord injury (iSCl) is influenced by spinal and supraspinal networks. Conventional clinical gait analysis fails to differentiate between these components. There is evidence that corticospinal control is enhanced during targeted walking, where each foot must be continuously placed on visual targets in randomized order. This study investigates the potential of targeted walking in the functional assessment of corticospinal integrity. Twenty-one controls and 16 individuals with chronic iSCl performed normal and targeted walking on a treadmill while electromyograms (EMGs) and kinematics were recorded. Precision (\% of accurate foot placements) in targeted walking was significantly lower in individuals with iSCl (82.9 $\pm 14.7 \%$, controls: $94.9 \pm 4.0 \%)$. While the overall kinematic pattern was comparable between walking conditions, controls showed significantly higher semitendinosus activity before heel-strike during targeted walking. This was accompanied by a shift of relative EMG intensity from $90-120 \mathrm{~Hz}$ to lower frequencies of $20-60 \mathrm{~Hz}$, previously associated with corticospinal control of muscle activity. Targeted walking in iSCl individuals evoked smaller EMG changes, suggesting that the switch to more corticospinal control is impaired. Accordingly, mildly impaired iSCl individuals revealed higher adaptations to the targeted walking task than more-impaired individuals. Recording of EMGs during targeted walking holds potential as a research tool to reveal further insights into the neuromuscular control of locomotion. It also complements findings of preclinical studies and is a promising novel surrogate marker of integrity of corticospinal control in individuals with iSCl and other neurological impairments. Future studies should investigate its potential for diagnosis or tracking recovery during rehabilitation.

Keywords: neuromuscular control, corticospinal control, targeted walking, locomotion, electromyography, corticospinal assessment 


\section{Introduction}

After incomplete spinal cord injury (iSCl), residual sensory and/or motor function below the lesion may allow full or partial recovery of locomotor function ${ }^{1,2}$. Locomotion is a complex motor task that is controlled by different neural systems including cortical and subcortical brain areas, spinal networks and afferent feedback ${ }^{3}$. It is assumed that relative cortical contribution to locomotion in humans compared to animals is phylogenetically greater due to demands of bipedal gait ${ }^{4,5}$.

Clinical walking tests and gait analysis of ordinary walking are used to assess locomotor function and rehabilitation outcomes after iSCl. These assessments are, however, unable to separately investigate spinal and supraspinal aspects contributing to deficits and recovery ${ }^{6}$. Assessments with systematic demands on different aspects of locomotor control promise better characterization and monitoring of deficits and treatment effects. In preclinical studies, it has been shown that the contribution of the cortex during locomotion is increased during anticipatory locomotor adaptations to imposed, external demands requiring visual integration and that this adaptability is decreased after impairments of corticospinal structures (e.g. iSCl or motor cortex lesions) ${ }^{7-10}$. Similarly, increased corticospinal control (input from cortical centers on spinal networks via the corticospinal tract) to locomotion has been shown in humans during anticipatory gait adaptations in visuo-motor targeted walking paradigms, in which foot placement is guided by randomized visual cues ${ }^{11-13}$. Targeted walking thus represents a suitable task for the assessment of corticospinal control of locomotion in humans.

Gait adaptations in such a task are achieved by sensory integration and resultant changes in muscle recruitment via descending pathways ${ }^{14,15}$, which can be quantified using electromyograms (EMGs). In cats, targeted walking was associated with enhanced activation of the semitendinosus (ST) muscle involved in controlling hindpaw placement at the end of swing phase, which disappeared after spinal cord hemisection and did not return despite 6 weeks of retraining, suggesting a supraspinal origin specific to targeted walking ${ }^{9}$. 
EMG time-frequency characteristics have been used to explore neural control mechanisms of muscle activity by analyzing temporal aspects of motor unit action potentials ${ }^{16}$. EMG activity within the range of $20-60 \mathrm{~Hz}$ (corticospinal drive frequencies) has been associated with corticospinal drive when investigated with corticomuscular and intermuscular coherence analysis $^{17-20}$. Increased synchrony, suggesting common neural input, or shifts in relative intensity of EMGs to corticospinal drive frequencies, have been observed during voluntary gait pattern modifications ${ }^{12,21}$, including targeted treadmill walking ${ }^{13}$. In contrast, decreased synchrony has been observed during voluntary movement in various neurological disorders ${ }^{22,23}$, including $\mathrm{iSCl}^{24}$. These findings suggest that $\mathrm{EMG}$ frequency characteristics may represent a useful tool for discerning cortical contribution to dynamic muscle activity during gait. In dynamic movements, EMG frequency characteristics can be investigated using intensity analysis in time-frequency space (e.g. wavelet analysis) ${ }^{25}$.

In this study, we build on recent findings to evaluate if a treadmill-based targeted walking paradigm with EMGs could be used to assess integrity of corticospinal control in individuals with iSCl. We hypothesize that control participants show more pronounced adaptations to a targeted walking task than individuals with iSCl which will manifest in greater targeting precision. We further hypothesize that these adaptations lead to measurable modulations of EMG amplitude and frequency characteristics, specifically intensity shifts to corticospinal drive frequencies. It is further investigated how changes in muscle activity are associated with kinematic adaptations to targeted walking.

\section{Materials and methods}

This study was approved by the cantonal ethics committee of Zurich, Switzerland (KEK-ZH 2014-0004) and completed according to Good Clinical Practice (GCP) guidelines and the Declaration of Helsinki. All participants gave informed, written consent.

\section{Participants}

Individuals with chronic (>12 months since injury) iSCl were recruited at University Hospital Balgrist in Zurich, Switzerland, while control participants were recruited from the local area via flyers. Control participants underwent detailed medical screening and were excluded if 
they had neurological or musculoskeletal disorders which could confound movement analysis. Control participants were matched to the iSCl group (age, sex, weight and height) from a large pool of control participants $(n=121)$. All individuals with iSCl had to be able to walk on a treadmill without the use of handrails.

\section{Experimental setup}

All study assessments were performed at the gait laboratory of the University Hospital Balgrist. Kinematic data were recorded with infrared cameras (Vicon UK) sampling at 200 $\mathrm{Hz}$. A set of 44 reflective markers was attached to each participant according to the Plugin-Gait model with additional technical locations ${ }^{26}$. Surface EMGs were recorded bilaterally with wireless transmitters (Noraxon Inc., Scottsdale, AZ, USA and myon AG, Switzerland; $10 \mathrm{~Hz}$ high-pass hardware filter; consistent transmitters within each participant) on the vastus medialis (VM), tibialis anterior (TA), gastrocnemius medialis (GM) and ST muscles according to the European recommendations for surface electromyography (SENIAM ${ }^{27}$ ). These muscles were selected due to their contributions to sagittal angular excursions during locomotion and their antagonistic relationship. EMGs were recorded at $1000 \mathrm{~Hz}$ and synchronized to the kinematic data (correcting wireless transmitter delays). All walking tasks were performed on a treadmill including a projector (Zebris FDM-T), which allowed the projection of target crosses onto the moving treadmill belt (Fig. 1A).

\section{Experimental design}

Assessments were performed in two sessions within one week. In a first session, all participants underwent a neurological examination by a physician to ensure that inclusion and exclusion criteria were met and to classify the neurological impairments in the iSCl group, which included the American Spinal Injury Association impairment scale (AIS) ${ }^{28}$ and the assessment of lower limb vibration sensation with a tuning fork at 4 locations (anterior superior iliac spine, patella, medial malleolus and dorsum of interphalangeal joint hallux), as a measure of proprioceptive function ${ }^{29}$. Participants were then familiarized with treadmill walking and the targeted walking task, while they were secured with a nonweight-bearing security harness that did not impair natural movement. Treadmill speed was set at $50 \%$ of the individual maximal walking velocity $\left(\mathrm{V}_{50 \% \max }\right)$, determined with the 
timed 25-foot walk test (T25FW, no walking aids or orthoses) and rounded to intervals of $0.3 \mathrm{~km} / \mathrm{h}$ beginning at $0.4 \mathrm{~km} / \mathrm{h}$ (i.e. $0.4 \mathrm{~km} / \mathrm{h}, 0.7 \mathrm{~km} / \mathrm{h}, 1 \mathrm{~km} / \mathrm{h}$ etc.). This relative speed was chosen to challenge participants on an equal level according to locomotor function and it corresponded to a comfortable walking speed. The targeted walking task involved the projection of crosses $(10 \times 10 \mathrm{~cm})$ onto the treadmill belt (Fig. 1A). The distance between the crosses on the treadmill was adjusted to the maximal step length and width of each participant, performed in a measurement of about 10 "maximally long and wide" steps. Crosses were projected in a randomized order (identical between participants) with an interval distance of $40-80 \%$ of maximal step length/width around a mean of $60 \%$. Approaching crosses were visible one step in advance. In total, all participants walked in the familiarization session (session 1) for at least 40 minutes on the treadmill, including performance of the targeted walking task for around 1 minute. In session 2 , an additional familiarization of at least 6 minutes treadmill walking was performed ${ }^{30}$. Afterwards, kinematic data and EMGs of 30-45 seconds normal treadmill walking and 1 minute targeted walking at $V_{50 \% \max }$ were recorded resulting in a minimum of approximately 25 strides (gait initiation and termination taking place before and after recording). Normal walking consisted of free treadmill walking while focusing on a cross on a screen in front of the treadmill. No projection on the moving belt was provided during the normal walking condition. Individuals with iSCl did not use walking aids, orthoses or the handrails in both conditions. However, some individuals were dependent on walking aids in everyday life. For the targeted walking task, participants were instructed to place the toe markers (on the second metatarsal head) as accurately as possible onto the middle of the crosses with each step, without endangering themselves. The targeted walking task was documented with a digital video camera $(30 \mathrm{~Hz})$ for evaluation of stepping precision. A 6-minute walk test (6MWT) was conducted at the end of session 2 as a quantification of community walking ability. Individuals with iSCl used their regular walking aids during the test.

\section{Data processing and statistical analysis}

Precision in the targeted walking task was evaluated manually from the video recordings. The criterion for an accurate placement was met when the center of a projected cross was hit by any part of a foot after contact of the whole foot (foot flat). The number of crosses 
accurately hit across the duration of the task is reported as a percentage of all crosses presented during the targeted walking task.

Gait events were identified from the kinematic data and defined as zero crossings of the heel (heel-strike) or toe marker velocity (toe-off) ${ }^{31,32}$. Kinematic and EMG data were normalized to the gait cycle (heel-strike to heel-strike, 0-100\%), stance phase (heel-strike to toe-off, 0-60\%) and swing phase (toe-off to heel-strike, 0-40\%). For control participants, data of the left and right side were analyzed separately. For individuals with iSCl, data were analyzed on the more (MI) and less impaired (LI) side, determined according to AIS motor (first criterion) or sensory (second criterion: light touch; third criterion: pin prick) scores. Stumbling steps (e.g. correction steps due to loss of balance in patients or adjustment of foot placement after initial foot strike) were visually identified using kinematic data and video recordings and discarded from all analyses (except for precision).

EMGs were bandpass-filtered ( $4^{\text {th }}$ order Butterworth, recursive) with cut-off frequencies of $10 \mathrm{~Hz}$ and $500 \mathrm{~Hz}$. After step normalization, every step was visually inspected and, in case of periods of signal loss, excluded for further EMG analysis. Root mean square (RMS) values were calculated for the swing and stance phase for each step cycle and muscle. To calculate group means and for visualization purposes, EMGs for each participant were smoothed with a moving average filter (window size $20 \mathrm{~ms}$ ) and amplitudes of both walking conditions were normalized to the $95^{\text {th }}$ percentile intensity during normal walking before averaging over the groups.

The EMG intensity of the filtered but non-normalized data of every step was decomposed into time and frequency components using a nonlinear wavelet transformation described in detail by von Tscharner ${ }^{25}$ and applied to the analysis of dynamic movements in various settings ${ }^{13,33}$. Briefly, the wavelet transformation results in the EMG intensity (square root of power) of every step being distributed across nine frequency bands around center frequencies 7, 19, 38, 62, 92, 128, 170, 218 and $271 \mathrm{~Hz}$. The lowest frequency band was affected by the hardware filter in this study and interpretation in this band is therefore limited. After wavelet transformation, EMG intensity in the frequency bands was time normalized to a gait cycle as mentioned above and averaged across all steps. For averaging 
over the whole group, EMG intensity in both walking condition was normalized to the $95^{\text {th }}$ percentile intensity during normal walking for each individual. The resulting muscle activity patterns are represented as heatmaps with time (\% of gait cycle) on the $\mathrm{x}$-axis, frequency band on the $y$-axis and the EMG intensity color-coded. To quantify the amount of EMG activity in a frequency band in a certain time window, EMG intensity can be summed over a period of the gait cycle. Summation in each band separately results in a discrete wavelet intensity spectrum, which includes the intensity quantification of the chosen period of the gait cycle distributed across the nine frequency bands. In order to make wavelet spectra independent from EMG intensity and consequently allow the comparison between participants and the two walking conditions, relative intensity in each frequency band is calculated as a percentage of the total intensity (sum of intensity in all frequency bands). A higher relative amount of EMG activity in the $38 \mathrm{~Hz}$ band has been associated to increased corticospinal control demands ${ }^{13}$. To compare frequency characteristics of specific muscle activity and not noise, wavelet spectra were calculated for windows of physiological muscle activity within stance (0-60\%) and swing (60-100\%), where muscles were active in both walking conditions. The windows were determined manually according to the group average heatmaps for controls and separately for each iSCl individual. Of the measured muscles, TA is the only one exhibiting a two peak activity during one of the considered phases. Previous studies have shown differences in cortical contribution to the two peaks of TA activity during the swing phase ${ }^{13,34}$. Consequently, the two peak activity of TA during swing was analyzed in two separate windows. These criteria resulted in the wavelet spectra of the following physiological muscle activities during normal and targeted walking in control participants and similar individually adjusted windows for each iSCl individual: VM stance: $1-20 \%$ of the gait cycle; VM swing: $85-100 \%$; TA stance: $1-30 \%$; TA swing 1: 60-80\%; TA swing 2, 85-100\%; GM stance: $10-50 \%$, ST stance: $1-10 \%$ and ST swing: 80-100\%.

To investigate if changes in relative EMG intensity in frequency bands were associated with different impairment levels after iSCl, the difference between the two walking conditions was calculated for individuals with iSCl for the frequency showing the greatest change in control participants $(38 \mathrm{~Hz})$. This difference was compared to the delta in control 
participants (control response). Furthermore, we investigated correlation of the difference in the $38 \mathrm{~Hz}$ band with clinical scores describing the impairment level of individuals with iSCI (6MWT, accuracy in targeted walking, light touch, LEMS, pin prick, vibration sensation score).

Kinematic data were processed with Vicon Nexus 2.5 (Vicon, Oxford, UK). Markers were labelled according the Plug-in-Gait model and gaps in the trajectories filled with appropriate algorithms provided by the software. Data were filtered with a Woltring filter with a volume-specific mean-square-error value of 15 and modelled with the Plug-in-Gait model. Data were then extracted to Matlab (Matlab 2017b, Mathworks Inc., Natick, USA). Sagittal angles for the lower limb joints were calculated with a vector-based approach using the marker positions of the toe, ankle, knee and the sacrum as well as the modelled hip joint rotation center. After normalization, range of motion (ROM) was calculated for every participant for swing and stance. Endpoint trajectories were analyzed using the anterior-posterior and vertical position of the toe markers. Vertical zero position of the marker was set to marker height at normal stance and the anterior-posterior position was normalized to the sacrum marker.

Statistical tests were performed using SPSS (V25, SPSS Inc., CA, USA), Matlab (Matlab 2017b, Mathworks Inc., Natick, MA, USA) and GraphPad Prism (V7, GraphPad Software, Inc., CA, USA). As precision values were normally distributed (Kolmogorov Smirnov, Shapiro-Wilk tests and visual inspection), the two groups were compared using an independent t-test, which was also used to compare the more- and less-impaired legs of individuals with iSCl. The influence of AIS motor and sensory scores on precision were investigated for each leg separately using Spearman correlations (one-sided), due to the ordinal scale of the measures.

Because RMS, wavelet spectra and ROM datasets were not all normally distributed, the normal and targeted walking conditions were compared using Wilcoxon signed-rank tests with Bonferroni correction for multiple comparisons across each group (corrected for number of muscles and side for RMS and ROM; for number of frequency bands in wavelet spectra). The delta between the walking conditions of relative EMG intensity in the band 
with the most prominent change $(38 \mathrm{~Hz})$ was normally distributed and therefore compared between groups using an ANOVA. Pearson (for continuous outcomes with normal distribution: age and 6MWT accuracy in targeted walking) and spearman correlation (for ordinal or non-normally distributed outcomes: AIS light touch, lower extremity motor score (LEMS), pin prick score, vibration sensation score and accuracy in \% during targeted walking) were used to investigate the relationship between this delta and variables describing the impairment level and the possible confounder age.

\section{Subgroup analysis}

The applied speed normalization resulted in different absolute walking speeds. To investigate the influence of walking speed on EMG patterns, we performed a subgroup analysis with a reduced dataset involving only the 6 fastest-walking individuals with iSCl and the 7 slowest control participants (several individuals walking at identical speeds resulted in a larger subgroup for control participants). Given the relative speed $\left(\mathrm{V}_{50 \% \text { max }}\right)$, these participants exhibited equal maximal walking speeds determined in the T25FW. RMS and the delta of relative EMG intensity in the $38 \mathrm{~Hz}$ band were compared between the subgroups using Mann-Withney-U-tests. Given the small sample size in the subgroups, the values for the two legs were combined in both cohorts for the statistical analysis.

\section{Results}

\section{Study population}

Control participants and individuals with iSCl did not differ significantly in terms of demographic or physical characteristics, while individuals with iSCl showed the expected neurological deficits and walked slower (Table 1). For the control group, EMG of 3 VM, 1 TA and $1 \mathrm{GM}$ were excluded due to inadequate data quality (absent signals due to technical issues or EMG saturation in the majority of steps). One iSCl participant was excluded from the precision analysis because of technical problems with the video recordings. 


\section{Precision}

Individuals with iSCl had significantly shorter maximal step length than controls and, therefore, performed the targeted walking task with shorter target distances (Table 1; independent $t$-test, $t(35)=3.86, p<0.001)$. Stepping precision was significantly decreased in individuals with iSCl compared to control participants $(t(34)=-3.58, p=0.001$, one individual with iSCl excluded due to technical problems with video recordings). There was no significant difference between the more and less impaired leg of individuals with iSCl (independent t-test, $t(28)=-0.78, p=0.44)$. Precision in the iSCl group was positively correlated with components of the AIS sensory scores (vibration: MI: $r=0.51, \mathrm{n}=15$, $p=0.026$, LI: $r=0.34, \mathrm{n}=15, p=0.108$; pin prick: MI: $r=0.64, \mathrm{n}=15, p=0.001$, LI: $r=-0.05, \mathrm{n}=15$, $p=0.431$; light touch: MI: $r=0.45, \mathrm{n}=15, p=0.046, \mathrm{LI}: r=0.29, \mathrm{n}=15, p=0.142$ ) but not the AIS LEMS (MI: $r=0.39, \mathrm{n}=15, p=0.075, \mathrm{LI}: r=0.16, \mathrm{n}=15, p=0.291$ ).

\section{EMG amplitude and activity pattern}

Control participants exhibited changes in muscle activity during the targeted walking task compared to that observed during normal walking (Fig. 1B \& C). These adaptations were similar irrespective of body side. During targeted walking, VM was more active during swing (Wilcoxon signed-rank test, $r V M$ swing: $Z=3.85, p<0.008, n=20, r=0.61$; IVM: $Z=2.94$, $p=0.021, n=19, r=0.48$ ) and stance ( $r V M$ stance: $Z=2.61, p=0.072, n=20, r=0.41$; IVM: $\mathrm{Z}=3.34, \mathrm{p}=0.008, \mathrm{n}=19, \mathrm{r}=0.54)$ compared to normal walking. Increased VM activity occurred around heel-strike (i.e. in late swing and early stance phase). Compared to normal walking, increased TA activity was only seen during the stance phase of targeted walking (rTA swing: $Z=0.93, p=1.0, n=20, r=0.15$; ITA swing: $Z=0.99, p=1.0, n=21 r=0.02$; $r$ A stance: $Z=3.85, p<0.008, n=20, r=0.61$; ITA stance: $Z=4.02, p<0.008, n=21, r=0.62)$. This increase was caused by a prolongation of TA activity beyond heel-strike without an increase in peak activity. GM exhibited a small, unilaterally (right side) significant increase in muscle activity during stance and a bilaterally significant increase during swing (rGA swing: $Z=3.98, p<0.008, n=21, r=0.61$; IGA swing: $Z=4.02, p<0.008, n=21, r=0.62 ; r G A$ stance: $Z=3.84, p<0.008, n=21, r=0.59$; IGA stance: $Z=2.49, p=0.104, n=21, r=0.38)$. This was driven by earlier onset of muscle activity following heel-strike and a newly-occurring, 
second burst during swing just before heel-strike. ST showed significantly increased activity during targeted walking in swing and stance ( $r S T$ swing: $Z=4.02, p<0.008, n=21$, $r=0.62$; IST swing: $Z=3.98, p<0.008, n=21, r=0.61$; $r S T$ stance: $Z=4.02, p<0.008, n=21$, $r=0.62$; IST stance: $Z=3.70, p<0.008, n=21, r=0.57)$. ST activity during stance was consistently higher during targeted versus normal walking. The increase during swing was characterized by a markedly higher peak muscle activity prior to heel-strike $(80 \%-100 \%$ of the gait cycle).

Individuals with iSCl showed a lower amount of significant changes in muscle activity in response to the targeted walking task (Fig. 1B \& D). The activity of VM around heel-strike was increased during targeted walking, similar to that observed in control participants (MI VM swing: $Z=2.69, p=0.056, n=16, r=0.47$; LI VM swing: $Z=2.79, p=0.040, n=16, r=0.49 ; M I$ VM stance: $Z=3.31, p=0.008, n=16, r=0.10$; LI VM stance: $Z=2.02, p=0.352, n=16, r=0.36$ ). Significantly increased TA and GM activity during stance was only present on the LI side (MI TA stance: $Z=2.54, p=0.088, n=16, r=0.65$; LI TA stance: $Z=3.10, p=0.016, n=16, r=0.55$ ). Analogous to controls, GM showed a newly occurring activity before heel strike (MI GM swing: $Z=3.05, p=0.016, n=16, r=0.54$; LI GM swing: $Z=3.52, p<0.008, n=16, r=0.62)$. Furthermore, a prolongation of ST activity after heel-strike resulted in significantly higher RMS values during the stance phase of targeted walking (MI ST stance: $Z=3.10, p=0.016$, $n=16, r=0.55$; LI ST stance: $Z=3.10, p=0.016, n=16, r=0.55)$.

\section{Kinematics}

Control participants showed kinematic adaptions which were also similar between the two body sides (Fig. 2A, C-E). During targeted walking, ROM was decreased for all leg joints (swing > stance, Fig. 2D). In the hip joint, this was caused by a reduced hip extension at toe-off during targeted compared to normal walking (Fig. 2A \& D). The knee was more flexed at heel-strike and during stance, while peak flexion during swing was reduced (Fig, $2 A)$. At the ankle, compared to normal walking, targeted walking was associated with less plantarflexion at toe-off but more around heel-strike, while dorsiflexion in early stance was increased (Fig. 2A). 
In contrast, individuals with $\mathrm{iSCl}$ showed neither a significant change in hip extension at toe-off nor altered ankle or hip ROM during stance or swing compared to normal walking $(2 B \& D)$. In the knee, similar changes were observed in the iSCl group to those in the control group (2B \& D). Besides more dorsiflexion at heel-strike and an earlier onset of plantarflexion before toe-off in the $\mathrm{MI}$ leg, ankle joint excursion was unchanged during targeted compared to normal walking in individuals with iSCl.

Whereas control participants performed shorter steps during targeted walking than in normal walking (paired t-test; right: $-12.8 \%, t(20)=5.252, p<0.002, n=21, r=0.76$; left: $10.9 \%, \mathrm{t}(20)=-5.104, \mathrm{p}<0.002, \mathrm{n}=21, \mathrm{r}=0.75)$, the $\mathrm{iSCl}$ group maintained their step length (MI: +5.6\%, t(15)=-1.699, $p=0.220 \mathrm{n}=16, \mathrm{r}=0.40 ; \mathrm{LI}:+5.9 \%, \mathrm{t}(15)=-1.945, p=0.14, \mathrm{n}=16$, $r=0.45)$. Control participants decreased their toe height in the early swing phase and at heel-strike during targeted walking, while increasing toe height during mid and late swing phase compared to normal walking. During targeted walking, the iSCl group only showed increased toe height in mid and late swing phase compared to normal walking but no changes of toe height in early swing phase and at heel-strike (Fig. 2C).

\section{EMG frequency characteristics}

Non-linear wavelet transformation revealed changes in the relative contribution of muscle activation frequencies at distinct points in the gait cycle during targeted walking in control participants (Fig. 3A, B). Changes between walking conditions were statistically not different between the two body sides (Supplementary table $1 \& 2$ ); therefore only data of the right side are shown. In the stance phase, there was a significant increase of relative intensity in the $38 \mathrm{~Hz}$ frequency band during targeted walking in TA and GM. Additionally, GM showed significant increases in relative intensity in the lowest two frequency bands and decreases in intermediate frequency bands (92 \& $128 \mathrm{~Hz}$, Fig. 3B). ST showed a significant increase of relative intensity in the 7, 19 and $38 \mathrm{~Hz}$ band during targeted walking in the swing phase, whereas TA showed a significant increase in the $19 \mathrm{~Hz}$ in the late swing phase (TA swing 2). The increase of relative ST intensity in the $38 \mathrm{~Hz}$ band during the swing phase was the most prominent change in frequency characteristics between the two walking conditions (Fig. 3B, red arrow) and is also observable in the time-frequency- 
intensity heatmaps (Fig. 3A). This coincides with the increased activity of ST right before heel-strike during targeted walking.

Individuals with $\mathrm{iSCl}$ showed fewer and less pronounced changes in frequency characteristics of muscle activity (Fig. 4). During stance, the only significant changes were found in the GM of the $\mathrm{MI}$ side with significant increases in the highest two frequency bands $(218 \& 271 \mathrm{~Hz})$. In TA, there was a significant increase in relative intensity in the 62 $\mathrm{Hz}$ band on the $\mathrm{MI}$ side in the late swing phase during targeted walking. In the ST during swing, there was an increase in relative intensity in the $38 \mathrm{~Hz}$ band during targeted walking, which was only statistically significant on the LI side. The shift of frequency in ST muscle activity during the swing phase in the LI leg is observable in the time-frequencyintensity heatmaps. However, the delta of relative EMG intensity in the $38 \mathrm{~Hz}$ band between normal and targeted walking in the ST during the swing phase was on both sides statistically smaller than in controls (Kruskal-Wallis test: $H(3)=14.92, p<0.001 ; \mathrm{r}$ controls vs. MI iSCl: $Z=3.758, p<0.001, r=0.62$; $r$ controls vs. $\mathrm{LI}$ iSCl: $Z=2.429, p=0.045, r=0.40$; Fig. 5A).

\section{iSCl impairment level and subgroup analyses for speed}

For the whole iSCl group, the delta in relative intensity in the $38 \mathrm{~Hz}$ band in the ST during swing was significantly correlated with the performance in the 6MWT (MI \& LI), the accuracy in the targeted walking task (MI) and the clinical vibration score (MI) (Fig. 5B-D). No correlations with further clinical scores (AIS light touch, LEMS, pin prick) or the confounding factor age were observed. Furthermore, no correlation was found in the control group between the delta in relative intensity in the $38 \mathrm{~Hz}$ band in ST during swing and accuracy in the targeted walking task (Spearman-correlation; $r=-0.01, n=21, p=0.976$ ), 6MWT performance (Pearson-correlation: $r=0.03, n=21, p=0.9030$ ) or treadmill speed $(r=0.11, n=21, p=0.635)$.

To investigate effects of walking speeds on our findings, we performed a speed-matched subgroup analysis (Controls: $n=7,3.4 \pm 0.3 \mathrm{~km} / \mathrm{h}$; iSCl: $n=6,3.4 \pm 0.2 \mathrm{~km} / \mathrm{h}$ ). Speed and maximal step length were not significantly different in these two subgroups (Supplementary table 3), indicating that the targeted walking sequences were highly comparable between group. Findings of muscle activity adaptations were in line with the 
overall cohorts (Fig. 6A). Individuals with iSCl in this subgroup showed more pronounced changes in muscle activity than the whole iSCl cohort and were more comparable to the control group. However, there was only a trend towards an increase of the ST before heelstrike and it was significantly smaller than in the control participants (Fig. 6A-C, MannWithney-U-test: $U=27.00, p=0.003, r=-0.58)$. Control participants in the speed-matched subgroup also demonstrated a prominent increase of relative intensity in the $38 \mathrm{~Hz}$ band of ST during swing during targeted walking, which was significantly larger than the delta in individuals with iSCl (Fig. 6D, $U=39.00, p=0.020, r=-0.45$ ). In this speed-matched subgroup, control participants and individuals with iSCl did not show decreased step length during targeted compared to normal walking (Wilcoxon signed-rank tests; controls: right: $-3.9 \%$, $Z=-0.734, p=0.926, n=7, r=0.19$; left: $-1.5 \%, Z=-0.314, p=1.0, n=7, r=0.08$; iSCl: MI: $-4.0 \%$, $Z=-0.943, p=0.690, n=6, r=0.27 ; M I:-4.0 \%, Z=-0.943, p=0.690, n=6, r=0.27)$.

\section{Discussion}

This study builds upon previous findings on corticospinal control during targeted walking in controls $^{11-13}$ and applies the task to individuals with iSCl. While the overall kinematic gait pattern remained similar between walking conditions, control participants showed distinct adaptations of EMG patterns and frequency characteristics during targeted walking. As the targeted walking task depends on anticipatory adaptations to randomized visual cues, differences between normal and targeted walking are likely caused by enhanced corticospinal control of locomotion with considerable contribution of the primary motor cortex, as demonstrated previously in animals ${ }^{8,9}$ and humans ${ }^{11-13}$. Locomotor adaptations were impaired in individuals with iSCl and correlated with the respective impairment level in walking capacity and proprioception, probably reflecting patients' integrity of corticospinal control of locomotion.

\section{Corticospinal control during targeted walking in control participants}

The most prominent difference in control participants was an increased activity in the ST during the swing phase in targeted compared to normal walking. As the ST extends the hip and flexes the knee, this increased muscle activity was likely involved in decelerating and controlling leg movement during the pendulum swing and thereby controlling foot 
placement $^{9,35}$. Remarkably, this finding replicates in humans those of Escalona et al, who observed ST activation in the late swing phase during targeted walking in cats ${ }^{9}$.

Corticospinal contribution to these prominent changes in ST activation during swing is further supported by observations in the EMG time-frequency analysis in connection with findings of previous studies. The most notable shift, observed in ST but also in TA and GM, was an increase of relative intensity in the $38 \mathrm{~Hz}$ and also the $19 \mathrm{~Hz}$ bands during targeted compared to normal walking. This was accompanied by a decrease of relative intensity in high frequency bands, reproducing findings of a previous study and broadening these results to proximal muscles ${ }^{13}$. The $19 \mathrm{~Hz}$ band overlaps with and the $38 \mathrm{~Hz}$ band is within the frequency range linked to corticospinal drive $(20-60 \mathrm{~Hz}$, cortical drive frequencies), in which increased corticomuscular coherence during targeted walking ${ }^{12}$ and increased intermuscular coherence during gait adaptations ${ }^{21}$ have been observed. The results of this study further support the notion that intensity shifts to the $20-60 \mathrm{~Hz}$ range and not only increased synchrony indicate different corticospinal control demands with possibly higher contribution from the motor cortex ${ }^{13}$.

Vastus medialis also showed increased activity during swing, underlining that VM together with ST controls final foot position through co-contraction. All lower limb muscles exhibited increased muscle activity either directly after foot strike or during stance phase. This is most likely caused by more balance requirements during targeting or increased contribution of the stance leg to the reaching of the swing leg ${ }^{36,37}$. Increases in corticospinal drive frequencies during stance were present in TA and GM but much more subtle than during the swing phase in ST, possibly indicating different changes of corticospinal control. Since brainstem and spinal centers have an important role in adjustments of balance and postural control ${ }^{38-41}$, it is very likely that they are involved in adaptations during stance phase in targeted walking besides cortical centers. However, the applied EMG-based time-frequency analysis is only able to gauge the involvement of corticospinal control in visually-guided targeted walking and does not provide information on the contribution of alternative, locomotor-relevant neural systems (e.g. descending brainstem areas, spinal networks, sensory afferents). 
Whereas the overall kinematic pattern remained comparable between walking conditions, reduced ROM and altered toe height in swing during targeted walking likely represent anticipatory strategies to increase time to react or retain options during targeting. Reduced toe height before heel-strike was associated with increased plantarflexion during targeting, as participants tended to approach the cross with the forefoot. This is further demonstrated by an additional second burst of GM activity towards the end of swing phase during targeted walking. Consequently, the rollover process was less pronounced, which is in line with a reduced peak EMG activity in the TA muscle after heel-strike ${ }^{42}$.

Previous studies have reported that corticospinal projections are pronounced in the flexor TA and a prominent role of corticospinal control during locomotion has been demonstrated for this muscle $34,43,44$. The results of this study similarly implicate increased corticospinal control of TA around heel-strike during targeted walking. Nevertheless, our results suggest that corticospinal control is more increased in the flexor ST than in TA during our targeted walking task, in line with the reasoning that proximal muscles assist foot placement ${ }^{9,45}$. GM is a distal muscle which assists foot placement by influencing step length $^{46}$, however, our findings similarly demonstrate higher adaptations in the leg in swing than in the contralateral leg in stance phase. Other muscles that were not recorded in this study might also contribute substantially to adjustments during targeted walking. This includes hip flexors for the control of step length and hip abductors for step width ${ }^{47}$.

\section{Impaired corticospinal adaptations after iSCl}

Although control participants and individuals with iSCl were able to hit the majority of crosses, individuals with iSCI performed significantly worse, replicating results from animal experiments with similar tasks ${ }^{8,9}$. The poorer precision exhibited by iSCl patients may, on one hand, be caused by damage to descending motor tracts, leading to insufficient motor adaptation. On the other hand, damaged ascending pathways may provide insufficient feedback for sensory integration ${ }^{48}$. Individuals with iSCl with greater sensory deficits showed a trend towards worse targeting precision, highlighting the importance of somatosensory integration. No correlation was found between LEMS and precision, 
indicating that the two measures represent different aspects of motor performance as previously shown with LEMS and locomotion ${ }^{49}$.

Besides reduced precision, individuals with iSCl showed impaired adaptation in muscle activity patterns during targeted walking, presumably reflecting reduced corticospinal control abilities. The prominent increase in ST activity demonstrated by control participants during swing in targeted walking was impaired in individuals with iSCl. This result again echoes Escalona et al, where the putatively cortical ST activation prior to paw placement disappeared after cord hemisection ${ }^{9}$. The interpretation of impaired corticospinal adaptations is further supported by the specific reduction of the shift towards corticispinal EMG frequencies in our iSCl population, which was observed in control participants during targeted walking ${ }^{13}$. It is unclear whether this is related directly to reduced corticospinal control (damaged descending tracts or reduced sensory feedback for integration) or rather is due to corticospinal drive being already at maximum during normal walking in individuals with iSCl due to higher attentional demands required to compensate deficits ${ }^{50-52}$. Besides the reduction of corticospinal EMG frequency shifts, individuals with iSCl showed increased activity in $\mathrm{GM}$ during stance phase in the higher frequency bands in direct contrast to the changes observed in control participants. Less is known about higher frequency bands, but this may indicate that individuals with iSCl activate more fast motor units during targeted walking ${ }^{33,53}$, possibly representing a compensation strategy.

Our findings in EMG frequency characteristics suggest that changes in relative intensity of corticospinal drive frequencies during targeted walking may be a marker of the integrity of corticospinal control of locomotion, as has been observed for intermuscular coherence in these frequencies ${ }^{22-24,54}$. Less-impaired individuals showed greater increases in relative intensity of corticospinal drive frequencies during targeted walking than more-impaired individuals. This suggests that this shift might allow the distinction of different levels of corticospinal control and could be used for monitoring effects of rehabilitation and treatments on corticospinal control, which would be of high clinical relevance. Synchrony between muscles in corticospinal drive frequencies has already been shown to increase with training in individuals with $\mathrm{iSCl}$, suggesting the restoration of corticospinal drive ${ }^{55}$. 
Test-retest reliability of EMG frequency characteristics is adequate enough for assessments in rehabilitation settings ${ }^{56,57}$, however, as this study did not involve a direct measure of corticospinal control and interpretations of frequency shifts were only based on literature, the validity needs further investigations. Comparisons to more direct measurements of corticospinal control (e.g. transcranial magnetic stimulation, electroencephalography) are required to confirm our findings. Besides this, large cohort trials with different lesion severities and at different stages of rehabilitation are required to evaluate the sensitivity of EMG time-frequency measures to gauge corticospinal control and its possible inferences on community walking function. The marked increase of corticospinal control during targeted walking in controls further suggests that the task may represent a rehabilitation modality to challenge patients and perhaps promote corticospinal control ${ }^{9,58}$.

\section{Limitations}

The relatively good performance of individuals with iSCl during targeted walking despite presumed impaired corticospinal control suggests that they may compensate with alternative neuronal systems. Alternatively, this may relate to methodological limitations. We used a binary classification for precision as used in previous studies ${ }^{8,9,12}$, but it remains possible that the observed changes in gait control are responsible for precision on a higher level. This issue could also explain similar accuracy between the MI and $\mathrm{LI}$ leg in individuals with iSCl.

In wavelet analysis, fatigue has been associated with an increase in EMG intensities in lower frequency bands $(7-88 \mathrm{~Hz})^{59}$, an unlikely confounder for EMG results in this study as this was in line with the effect observed in the control group, not the iSCl group more susceptible to fatigue. However, fatigue might have negatively influenced concentration and consequently the accuracy performance. The length of the treadmill limited the maximal projection step length to $66 \mathrm{~cm}$ during targeted walking, leading to some participants walking with shorter steps than during normal walking. However, as control participants and individuals with iSCl of the speed-matched subgroups did not change the 
average step length between walking conditions, it can be concluded that the observed differences between groups are not caused by this issue.

\section{Conclusion}

Normal treadmill walking may not be demanding enough to allow the detection of differences in corticospinal control in ambulatory individuals with iSCl, potentially masking treatment and therapy effects and the distinction between spinal and supraspinal aspects of locomotion. By analyzing EMG patterns and frequency characteristics, this study identifies corticospinal control strategies in control participants during targeted walking, which were most prominently in ST. In chronic iSCl, these strategies appear to be disturbed and result in less precise foot placement. Targeted walking may hold potential as an investigative and rehabilitative locomotor modality for the detection and promotion of functional integrity of corticospinal control in individuals with iSCl and other neurological impairments, which should be further explored in future studies.

\section{Acknowledgements}

We thank all volunteers who kindly donated their time and energy to participate in this study. We thank Volker Dietz and Serge Rossignol for critical reading of the manuscript and for their comments that greatly improved the manuscript. This study was supported by the Clinical Research Priority Program for NeuroRehab of the University of Zurich. Movement analysis was supported by the Swiss Center for Clinical Movement Analysis, SCMA, Balgrist Campus AG, Zürich.

\section{Author Disclosure Statement}

The authors declare no competing financial interests 


\section{References}

1. Barbeau, H., Ladouceur, M., Norman, K.E., Pépin, A., and Leroux, A. (1999). Walking after spinal cord injury: Evaluation, treatment, and functional recovery. Arch. Phys. Med. Rehabil. 80, 225-235.

2. Ditunno, P.L., Patrick, M., Stineman, M., and Ditunno, J.F. (2008). Who wants to walk? Preferences for recovery after SCl: A longitudinal and cross-sectional study. Spinal Cord 46, 500-506.

3. Grillner, S. (2011). Control of Locomotion in Bipeds, Tetrapods, and Fish., in: Comprehensive Physiology. .

4. Drew, T., and Marigold, D.S. (2015). Taking the next step: Cortical contributions to the control of locomotion. Curr. Opin. Neurobiol. 33, 25-33.

5. De Leon, R.D., Hodgson, J.A., Roy, R.R., and Edgerton, V.R. (1998). Locomotor capacity attributable to step training versus spontaneous recovery after spinalization in adult cats. J. Neurophysiol. 79, 1329-1340.

6. Bolliger, M., Blight, A.R., Field-Fote, E.C., Musselman, K., Rossignol, S., Barthélemy, D., Bouyer, L., Popovic, M.R., Schwab, J.M., Boninger, M.L., Tansey, K.E., Scivoletto, G., Kleitman, N., Jones, L.A.T., Gagnon, D.H., Nadeau, S., Haupt, D., Awai, L., Easthope, C.S., Zörner, B., Rupp, R., Lammertse, D., Curt, A., and Steeves, J. (2018). Lower extremity outcome measures: Considerations for clinical trials in spinal cord injury. Spinal Cord 56, 628-642.

7. Farrell, B.J., Bulgakova, M.A., Sirota, M.G., Prilutsky, B.I., and Beloozerova, I.N. (2015). Accurate stepping on a narrow path: Mechanics, EMG, and motor cortex activity in the cat. J. Neurophysiol. 114, 2682-2702.

8. Zörner, B., Filli, L., Starkey, M.L., Gonzenbach, R., Kasper, H., Röthlisberger, M., Bolliger, M., and Schwab, M.E. (2010). Profiling locomotor recovery: Comprehensive quantification of impairments after CNS damage in rodents. Nat. Methods 7, 701708. 
9. Escalona, M., Delivet-Mongrain, H., Kundu, A., Gossard, J.P., and Rossignol, S. (2017). Ladder treadmill: A method to assess locomotion in cats with an intact or lesioned spinal cord. J. Neurosci. 37, 5429-5446.

10. Drew, T., Jiang, W., and Widajewicz, W. (2002). Contributions of the motor cortex to the control of the hindlimbs during locomotion in the cat. Brain Res. Rev. 40, 178191.

11. Schubert, M., Curt, A., Colombo, G., Berger, W., and Dietz, V. (1999). Voluntary control of human gait: Conditioning of magnetically evoked motor responses in a precision stepping task. Exp. Brain Res. 126, 583-588.

12. Jensen, P., Jensen, N.J., Terkildsen, C.U., Choi, J.T., Nielsen, J.B., and Geertsen, S.S. (2018). Increased central common drive to ankle plantar flexor and dorsiflexor muscles during visually guided gait. Physiol. Rep. 6.

13. Filli, L., Meyer, C., Killeen, T., Lörincz, L., Göpfert, B., Linnebank, M., Von Tscharner, V., Curt, A., Bolliger, M., and Zörner, B. (2019). Probing corticospinal control during different locomotor tasks using detailed time-frequency analysis of electromyograms. Front. Neurol. 10.

14. Lange, G.W., Hintermeister, R.A., Schlegel, T., Dillman, C.J., and Richard Steadman, J. (1996). Electromyographic and kinematic analysis of graded treadmill walking and the implications for knee rehabilitation. J. Orthop. Sports Phys. Ther. 23, 294-301.

15. Barbeau, H., Fung, J., Leroux, A., and Ladouceur, M. (2002). A review of the adaptability and recovery of locomotion after spinal cord injury., in: Progress in Brain Research. pps. 9-25.

16. Farina, D., Merletti, R., and Enoka, R.M. (2004). The extraction of neural strategies from the surface EMG. J. Appl. Physiol. 96, 1486-1495.

17. Brown, P. (2000). Cortical drives to human muscle: The Piper and related rhythms. Prog. Neurobiol. 60, 97-108. 
18. Marsden, J.F., Werhahn, K.J., Ashby, P., Rothwell, J., Noachtar, S., and Brown, P. (2000). Organization of cortical activities related to movement in humans. J. Neurosci. 20, 2307-2314.

19. Fisher, K.M., Zaaimi, B., Williams, T.L., Baker, S.N., and Baker, M.R. (2012). Betaband intermuscular coherence: A novel biomarker of upper motor neuron dysfunction in motor neuron disease. Brain 135, 2849-2864.

20. Jensen, P., Frisk, R., Spedden, M.E., Geertsen, S.S., Bouyer, L.J., Halliday, D.M., and Nielsen, J.B. (2019). Using Corticomuscular and Intermuscular Coherence to Assess Cortical Contribution to Ankle Plantar Flexor Activity During Gait. J. Mot. Behav. 51, $668-680$.

21. Clark, D.J., Kautz, S.A., Bauer, A.R., Chen, Y.T., and Christou, E.A. (2013). Synchronous EMG activity in the piper frequency band reveals the corticospinal demand of walking tasks. Ann. Biomed. Eng. 41, 1778-1786.

22. Nielsen, J.B., Brittain, J.S., Halliday, D.M., Marchand-Pauvert, V., Mazevet, D., and Conway, B.A. (2008). Reduction of common motoneuronal drive on the affected side during walking in hemiplegic stroke patients. Clin. Neurophysiol. 119, 28132818.

23. Velázquez-Pérez, L., Tünnerhoff, J., Rodríguez-Labrada, R., Torres-Vega, R., RuizGonzalez, Y., Belardinelli, P., Medrano-Montero, J., Canales-Ochoa, N., GonzálezZaldivar, Y., Vazquez-Mojena, Y., Auburger, G., and Ziemann, U. (2017). Early corticospinal tract damage in prodromal SCA2 revealed by EEG-EMG and EMG-EMG coherence. Clin. Neurophysiol. 128, 2493-2502.

24. Hansen, N.L., Conway, B.A., Halliday, D.M., Hansen, S., Pyndt, H.S., Biering-Sørensen, F., and Nielsen, J.B. (2005). Reduction of common synaptic drive to ankle dorsiflexor motoneurons during walking in patients with spinal cord lesion. J. Neurophysiol. 94, 934-942. 
25. Von Tscharner, V. (2000). Intensity analysis in time-frequency space of surface myoelectric signals by wavelets of specified resolution., in: Journal of Electromyography and Kinesiology. pps. 433-445.

26. Vicon. (2002). Plug-in-Gait modelling instructions. Plug-in-Gait Man. , 612.

27. Hermens, H.J., Freriks, B., Disselhorst-Klug, C., and Rau, G. (2000). Development of recommendations for SEMG sensors and sensor placement procedures. J. Electromyogr. Kinesiol. 10, 361-374.

28. Marino, R.J., Barros, T., Biering-Sorensen, F., Burns, S.P., Donovan, W.H., Graves, D.E., Haak, M., Hudson, L.M., and Priebe, M.M. (2003). International standards for neurological classification of spinal cord injury. J. spinal cord Med. 26 Suppl 1.

29. Martina, I.S.J., Van Koningsveld, R., Schmitz, P.I.M., Van Der Meché, F.G.A., and Van Doorn, P.A. (1998). Measuring vibration threshold with a graduated tuning fork in normal aging and in patients with polyneuropathy. J. Neurol. Neurosurg. Psychiatry $65,743-747$.

30. Meyer, C., Killeen, T., Easthope, C.S., Curt, A., Bolliger, M., Linnebank, M., Zörner, B., and Filli, L. (2019). Familiarization with treadmill walking: How much is enough? Sci. Rep. 9, 5232.

31. Zeni, J.A., Richards, J.G., and Higginson, J.S. (2008). Two simple methods for determining gait events during treadmill and overground walking using kinematic data. Gait Posture 27, 710-714.

32. Filli, L., Sutter, T., Easthope, C.S., Killeen, T., Meyer, C., Reuter, K., Lörincz, L., Bolliger, M., Weller, M., Curt, A., Straumann, D., Linnebank, M., and Zörner, B. (2018). Profiling walking dysfunction in multiple sclerosis: Characterisation, classification and progression over time. Sci. Rep. 8.

33. Von Tscharner, V., Ullrich, M., Mohr, M., Comaduran Marquez, D., and Nigg, B.M. (2018). A wavelet based time frequency analysis of electromyograms to group steps of runners into clusters that contain similar muscle activation patterns. PLoS One 13. 
34. Schubert, M., Curt, A., Jensen, L., and Dietz, V. (1997). Corticospinal input in human gait: Modulation of magnetically evoked motor responses. Exp. Brain Res. 115, 234246.

35. Arnell, P. (1988). The Biomechanics and Motor Control of Human Gait., in: Physiotherapy. pp. 94.

36. Arnold, A.S., Thelen, D.G., Schwartz, M.H., Anderson, F.C., and Delp, S.L. (2007). Muscular coordination of knee motion during the terminal-swing phase of normal gait. J. Biomech. 40, 3314-3324.

37. Cimadoro, G., Paizis, C., Alberti, G., and Babault, N. (2013). Effects of different unstable supports on EMG activity and balance. Neurosci. Lett. 548, 228-232.

38. Dietz, V., Trippel, M., and Horstmann, G.A. (1991). Significance of Proprioceptive and Vestibulo - Spinal Reflexes in the Control of Stance and Gait. Adv. Psychol. .

39. Deliagina, T.G., Orlovsky, G.N., Zelenin, P. V., and Beloozerova, I.N. (2006). Neural bases of postural control. Physiology .

40. Boisgontier, M.P., Cheval, B., Chalavi, S., van Ruitenbeek, P., Leunissen, I., Levin, O., Nieuwboer, A., and Swinnen, S.P. (2017). Individual differences in brainstem and basal ganglia structure predict postural control and balance loss in young and older adults. Neurobiol. Aging .

41. Dietz, V., and Duysens, J. (2000). Significance of load receptor input during locomotion: A review. Gait Posture.

42. Yong, J.R., Silder, A., and Delp, S.L. (2014). Differences in muscle activity between natural forefoot and rearfoot strikers during running. J. Biomech. 47, 3593-3597.

43. Brouwer, B., and Ashby, P. (1992). Corticospinal projections to lower limb motoneurons in man. Exp. Brain Res. 89, 649-654. 
44. Capaday, C., Lavoie, B.A., Barbeau, H., Schneider, C., and Bonnard, M. (1999). Studies on the corticospinal control of human walking. I. Responses to focal transcranial magnetic stimulation of the motor cortex. J. Neurophysiol. 81, 129-139.

45. Bruijn, S.M., and Van Dieën, J.H. (2018). Control of human gait stability through foot placement. J. R. Soc. Interface 15.

46. JudgeRoy, J.O., Davis, B., and Ounpuu, S. (1996). Step Length Reductions in Advanced Age: The Role of Ankle and Hip Kinetics. Journals Gerontol. Ser. A Biol. Sci. Med. Sci. .

47. Rankin, B.L., Buffo, S.K., and Dean, J.C. (2014). A neuromechanical strategy for mediolateral foot placement in walking humans. J. Neurophysiol. .

48. Ahuja, C.S., Nori, S., Tetreault, L., Wilson, J., Kwon, B., Harrop, J., Choi, D., and Fehlings, M.G. (2017). Traumatic spinal cord injury - Repair and regeneration. Clin. Neurosurg. 80, S22-S90.

49. Wirz, M., van Hedel, H.J., Rupp, R., Curt, A., and Dietz, V. (2006). Muscle Force and Gait Performance: Relationships After Spinal Cord Injury. Arch. Phys. Med. Rehabil. $87,1218-1222$.

50. Lajoie, Y., Barbeau, H., and Hamelin, M. (1999). Attentional requirements of walking in spinal cord injured patients compared to normal subjects. Spinal Cord 37, 245250.

51. Mihara, M., Miyai, I., Hatakenaka, M., Kubota, K., and Sakoda, S. (2007). Sustained prefrontal activation during ataxic gait: A compensatory mechanism for ataxic stroke? Neuroimage 37, 1338-1345.

52. Plotnik, M., Giladi, N., and Hausdorff, J.M. (2009). Bilateral coordination of gait and Parkinson's disease: The effects of dual tasking. J. Neurol. Neurosurg. Psychiatry 80, 347-350.

53. Wakeling, J.M., and Rozitis, A.I. (2004). Spectral properties of myoelectric signals from different motor units in the leg extensor muscles. J. Exp. Biol. 207, 2519-2528. 
54. Lodha, N., Chen, Y.T., McGuirk, T.E., Fox, E.J., Kautz, S.A., Christou, E.A., and Clark, D.J. (2017). EMG synchrony to assess impaired corticomotor control of locomotion after stroke. J. Electromyogr. Kinesiol. 37, 35-40.

55. Norton, J.A., and Gorassini, M.A. (2006). Changes in cortically related intermuscular coherence accompanying improvements in locomotor skills in incomplete spinal cord injury. J. Neurophysiol. 95, 2580-2589.

56. Van Asseldonk, E.H.F., Campfens, S.F., Verwer, S.J.F., Van Putten, M.J.A.M., and Stegeman, D.F. (2014). Reliability and agreement of intramuscular coherence in tibialis anterior muscle. PLoS One 9.

57. Coorevits, P., Danneels, L., Cambier, D., Ramon, H., Druyts, H., Karlsson, J.S., De Moor, G., and Vanderstraeten, G. (2008). Test-retest reliability of wavelet - and Fourier based EMG (instantaneous) median frequencies in the evaluation of back and hip muscle fatigue during isometric back extensions. J. Electromyogr. Kinesiol. $18,798-806$.

58. Yang, J.F., and Gorassini, M. (2006). Spinal and brain control of human walking: Implications for retraining of walking. Neuroscientist 12, 379-389.

59. Sparto, P.J., Parnianpour, M., Barria, E.A., and Jagadeesh, J.M. (2000). Wavelet and short-time fourier transform analysis of electromyography for detection of back muscle fatigue. IEEE Trans. Rehabil. Eng. 8, 433-436. 
Table 1: Group characteristics. Characteristics of the two populations (mean \pm SD or median \pm range for clinical scores). P-values are for statistical comparisons between the groups (independent t-test for age, height weight, 6MWT, speed, maximal step length, maximal step width, precision; Chi-square test for gender; Mann-Whitney U-test for motor score, sensory scores (light touch \& pinprick), vibration, position sense and modified Ashworth scale). 6MWT, 6-minute walking test; n/a, not applicable.

\begin{tabular}{|c|c|c|c|}
\hline & Control group & iSCl & p-value \\
\hline $\mathbf{n}$ & 21 & 16 & \\
\hline Gender [female : male] & $9: 12$ & $5: 11$ & 0.47 \\
\hline Age [years] & $53.4 \pm 13.9$ & $51.7 \pm 8.6$ & 0.67 \\
\hline Height $[\mathrm{cm}]$ & $172.0 \pm 6.9$ & $167.3 \pm 30.2$ & 0.42 \\
\hline Weight [kg] & $70.3 \pm 9.9$ & $80.8 \pm 28.2$ & 0.46 \\
\hline 6MWT [m] & $721 \pm 87$ & $483 \pm 137$ & $<0.01$ \\
\hline $\begin{array}{r}50 \% \text { of maximal speed } \\
{[\mathrm{km} / \mathrm{h}]}\end{array}$ & $4.0 \pm 0.5$ & $2.7 \pm 0.7$ & $<0.01$ \\
\hline Maximal step length [cm] & $154.7 \pm 12.4$ & $125.5 \pm 31.8$ & $<0.01$ \\
\hline Maximal step width [cm] & $31.5 \pm 3.0$ & $30.3 \pm 5.0$ & 0.34 \\
\hline Precision [\%] & $94.9 \pm 4.0$ & $82.9 \pm 14.7$ & $<0.01$ \\
\hline AIS & $\mathrm{n} / \mathrm{a}$ & $D(16)$ & $\mathrm{n} / \mathrm{a}$ \\
\hline Lesion level & $\mathrm{n} / \mathrm{a}$ & $\begin{array}{l}\text { cervical (7) } \\
\text { thoracic (9) }\end{array}$ & $\mathrm{n} / \mathrm{a}$ \\
\hline
\end{tabular}




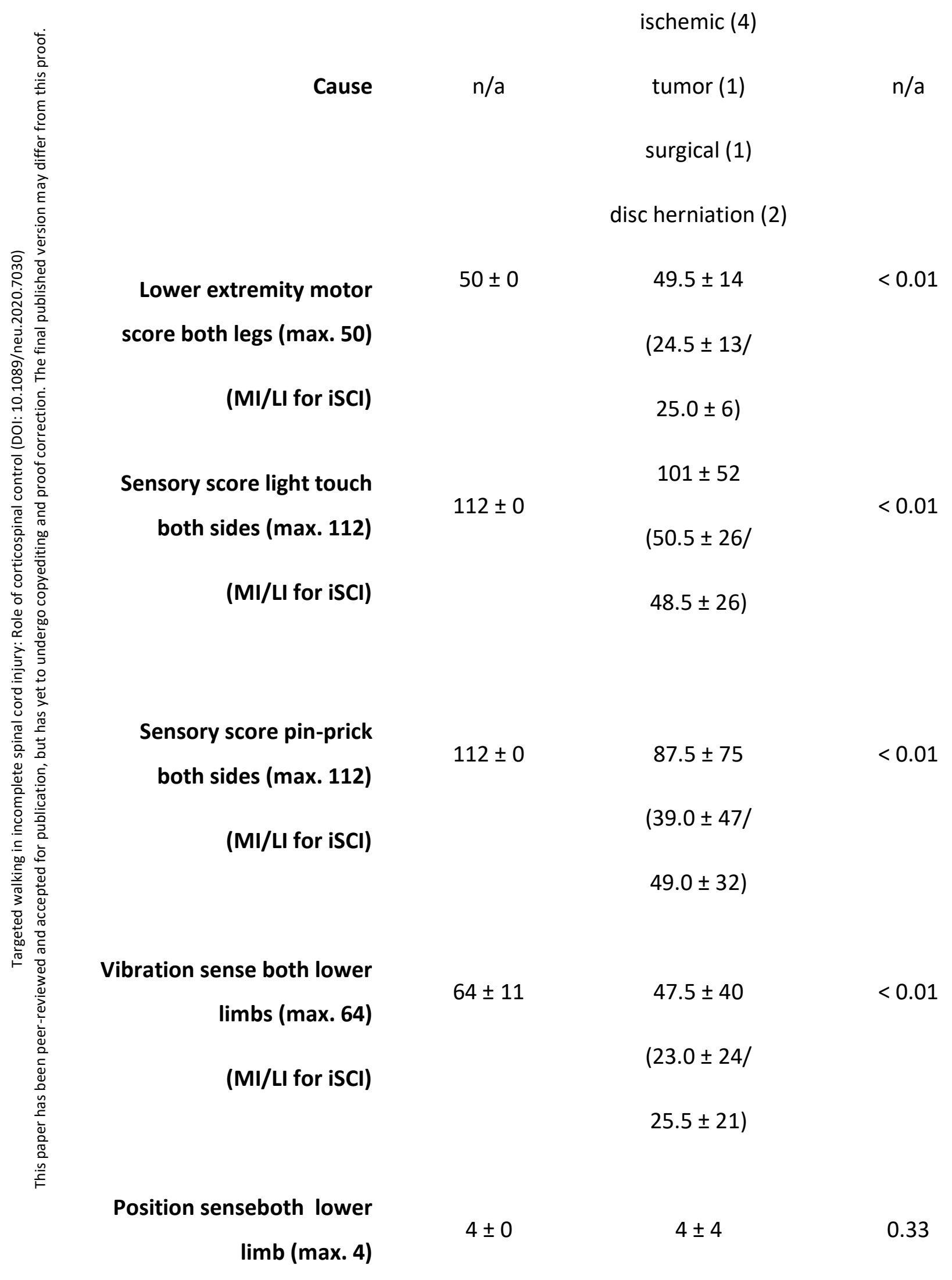

traumatic (8)

ischemic (4) 
(MI/LI for iSCl)

$$
(2 \pm 4)
$$

$2 \pm 4)$

$\begin{array}{rcc}\text { Modified Ashworth scale } & 0 \pm 7 & 0.06 \\ \text { both legs }(\max .16) & 0 \pm 0 & (0 \pm 7 /\end{array}$

(MI/LI for iSCl)

$$
0 \pm 2.5)
$$


A Figure 1

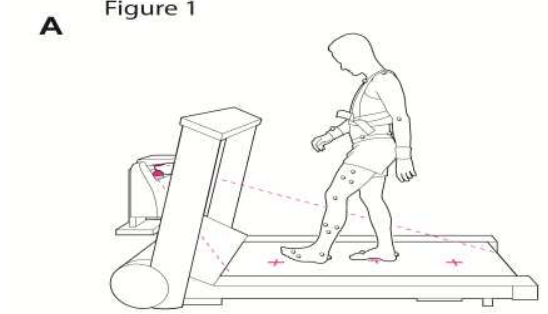

c

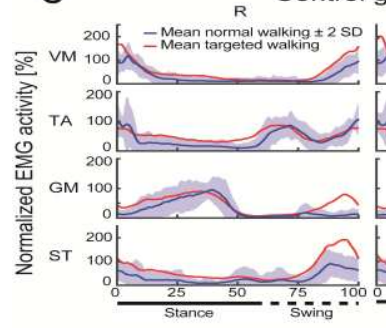

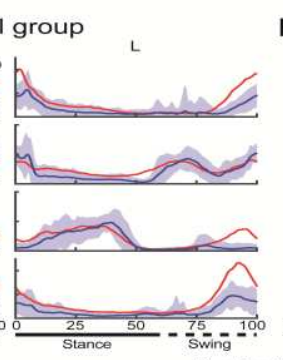

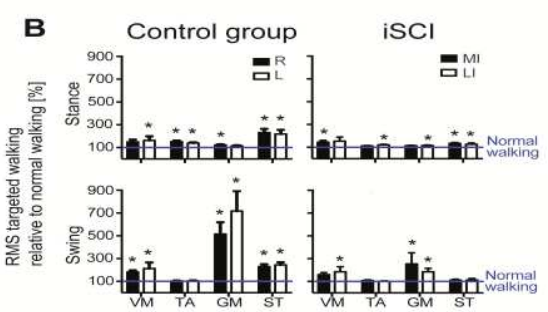

D

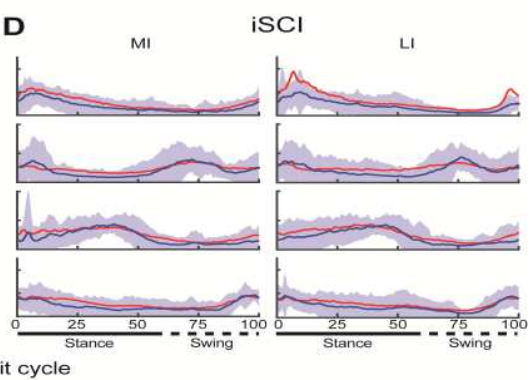

Figure 1: EMG patterns during normal and targeted walking in control participants and individuals with iSCl. A) Experimental setup (University of Zurich, Information Technology, MELS/SIVIC, Tara von Grebel). B) Mean power (RMS) of EMG activity for control participants and individuals with iSCl during the stance and swing phases (mean \pm standard error of the mean). The targeted walking condition is presented relative to normal walking EMG activity (blue line). Data for the control group right and left VM (r: $n=20 ; 1: n=19), T A$ $(r: n=20 ; I: n=21), G M(r: n=21 ; I: n=20)$ and ST (r: $n 21=; I: n=21)$ is shown together with the same muscles for the more and less impaired side of the individuals with iSCl $(n=16)$. Asterisks indicate a significant difference between normal and targeted walking $(\alpha=0.05)$ tested by Wilcoxon signed-rank tests with Bonferroni correction for multiple comparisons. C) Rectified and averaged EMG activity of control participants during normal (mean \pm 2 standard deviations) and targeted walking (mean) for right and left VM, TA, GM and ST (for $n$ see section $B)$. The EMG amplitudes were normalized to the $95^{\text {th }}$ percentile intensity during normal walking. D) Rectified and averaged EMG activity of individuals with iSCl ( $n=16)$ during normal (mean \pm 2 standard deviations) and targeted walking (mean) for more and less impaired VM, TA, GM, ST. The EMG amplitudes were normalized to the $95^{\text {th }}$ percentile intensity during normal walking. a.u., arbitrary unit; $\mathrm{iSCl}$, incomplete spinal cord injury; RMS, root mean square; R, right; L, left; MI, more impaired; LI, less impaired; SD, standard deviation; VM, vastus medialis; TA, tibialis anterior; GM, gastrocnemius; ST, semitendinosus. 

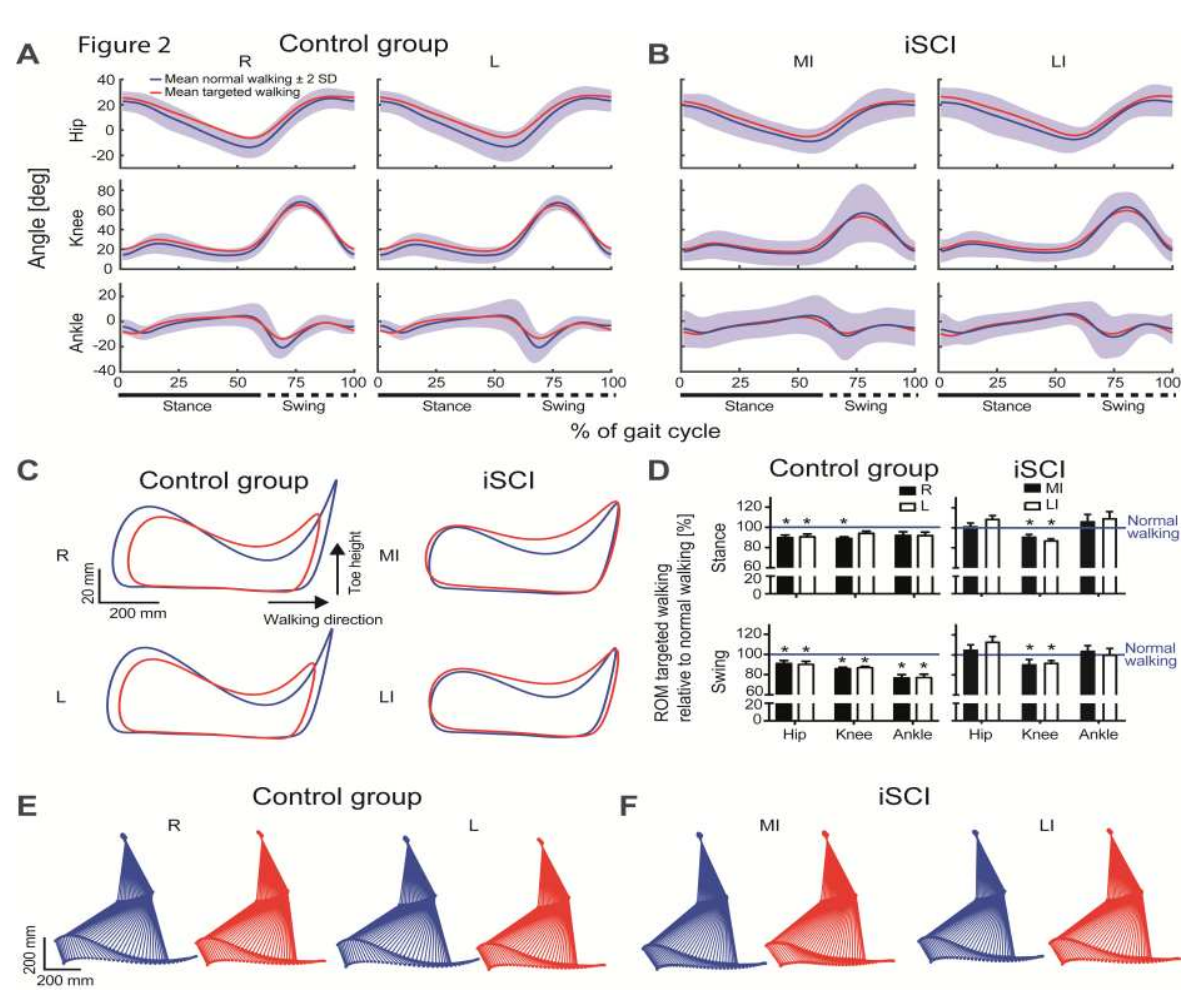

iscl

Figure 2: Kinematic patterns during normal and targeted walking in control participants and individuals with iSCI. A) Average sagittal joint angles of controls $(n=21)$ for right and left hip, knee and ankle during normal (blue, mean \pm 2 standard deviations) and targeted walking (red, mean). B) Average sagittal joint angles of individuals with iSCl ( $n=16)$ for more and less impaired hip, knee and ankle during normal (blue, mean \pm 2 standard deviations) and targeted walking (red, mean). C) 2D illustration of the average toe trajectories for control participants $(n=21)$ and individuals with iSCl $(n=16)$ during normal (blue) and targeted walking (red). The anterior-posterior position was normalized to the sacrum. D) ROM (mean \pm standard error of the mean) of controls $(n=21)$ and individuals with iSCl $(n=16)$ for hip, knee and ankle during the stance and swing phases. The targeted walking data is presented relative to normal walking, which is indicated by a blue line. Asterisks indicate a significant difference between normal and targeted walking ( $\alpha=0.05)$ tested by Wilcoxon signed-rank tests with Bonferroni correction for multiple comparisons. E) Stick figures during normal and targeted walking for the right and left leg of control participants. F) Stick figures during normal and targeted walking for the more and less impaired leg of individuals with iSCl. iSCl, incomplete spinal cord injury; R, right; L, left; $\mathrm{MI}$, more impaired; LI, less impaired; SD, standard deviation; ROM, range of motion. 
A Figure
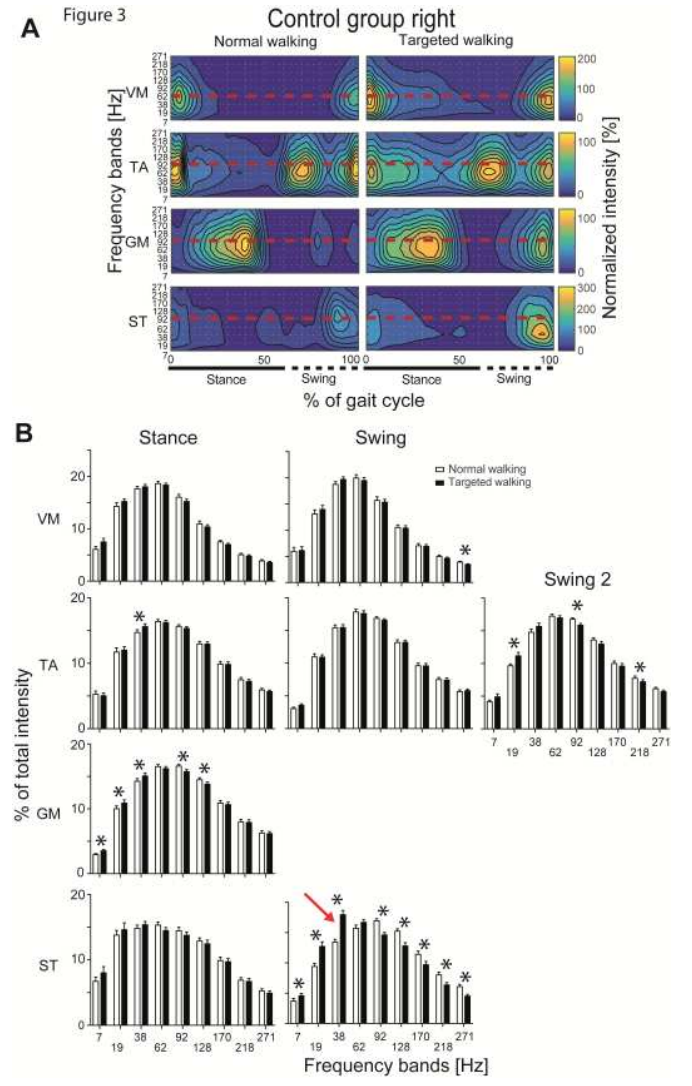

Figure 3: EMG frequency characteristics during normal and targeted walking in control participants. A) Average time-frequency-intensity heatmaps (wavelet transformation) for control participants right VM $(n=20), T A(n=20), G M(n=21)$ and ST $(n=21)$. The EMG intensity (amplitude) was normalized to the $95^{\text {th }}$ percentile intensity during normal walking (over all bands) for each individual. The color scale of the heatmap ranges from 0 to the maximal intensity of the 2 conditions for each muscle. The frequency band of the maximal intensity during normal walking is indicated for both conditions with a dashed red line. B) Average (mean \pm standard error of the mean) percentage distribution of EMG intensity across the 9 frequency bands (wavelet spectra) for physiological muscle activity within stance and swing phases (see methods) of control participants right VM $(n=20), T A(n=20)$, GM $(n=21)$ and ST $(n=21)$ during normal and targeted walking. Asterisks indicate a significant difference between normal and targeted walking $(\alpha=0.05)$ tested by Wilcoxon signed-rank tests with Bonferroni correction for multiple comparisons. The most prominent shift in intensity distribution is indicated with a red arrow. VM, vastus medialis; TA, tibialis anterior; GM, gastrocnemius; ST, semitendinosus. 


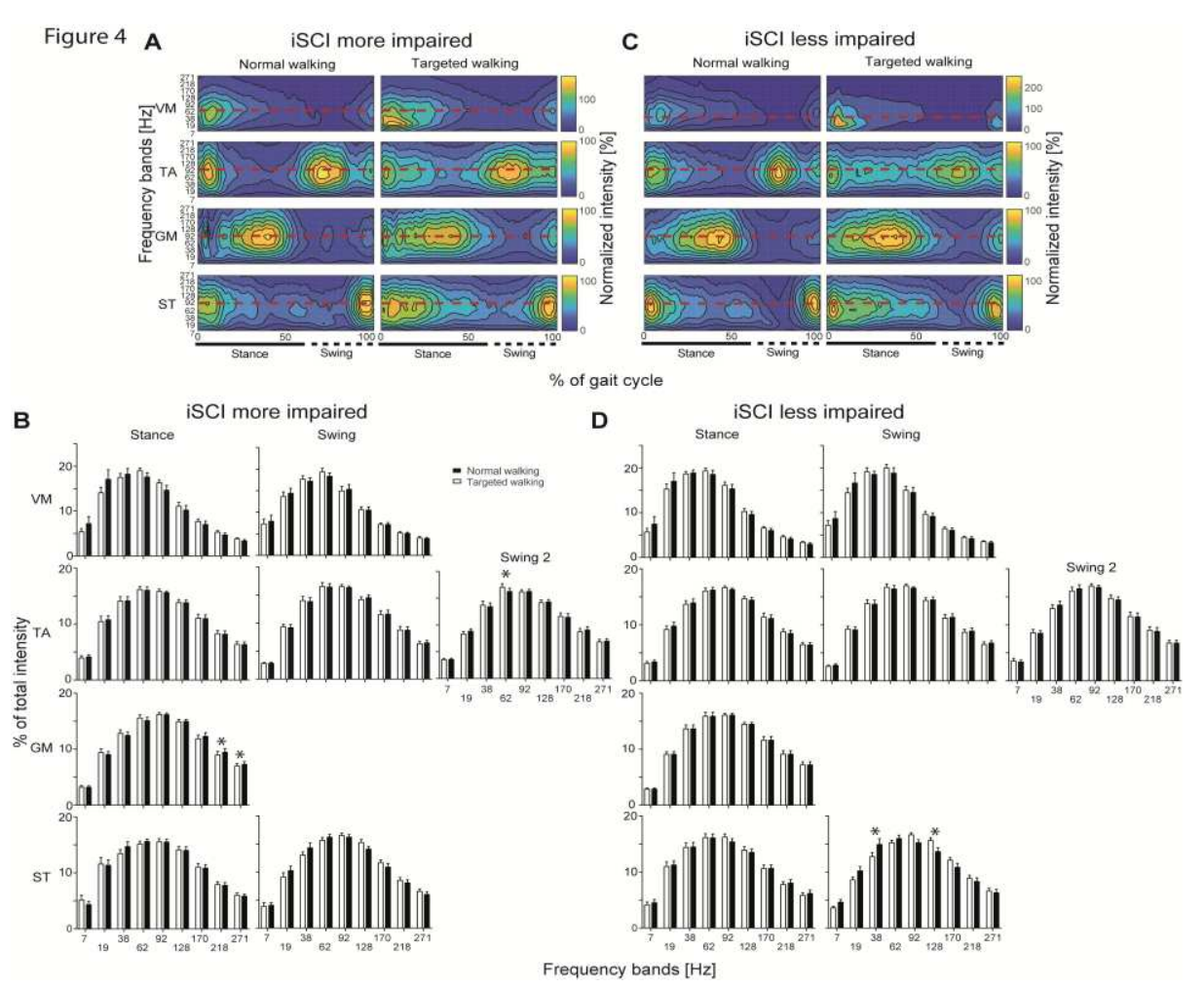

Figure 4: EMG frequency characteristics during normal and targeted walking in individuals with iSCI. A \& C) Average time-frequency-intensity heatmaps (wavelet transformation) for more (A) and less (C) impaired VM, TA, GM and ST of individuals with iSCI. The EMG intensity (amplitude) was normalized to the $95^{\text {th }}$ percentile intensity during normal walking for each individual. The color scale of the heatmap ranges from 0 to the maximal intensity of the 2 conditions for each muscle. The frequency band of the maximal intensity during normal walking is indicated for both conditions with a dashed red line. B \& D) Average (mean \pm standard error of the mean) percentage distribution of EMG intensity across the 9 frequency bands (wavelet spectra) for individual muscle activity phases within stance and swing (see methods) of more (B) and less (D) impaired VM, TA, GM and ST of individuals with iSCl $(n=16)$ during normal and targeted walking. Asterisks indicate a significant difference between normal and targeted walking $(\alpha=0.05)$ tested by Wilcoxon signed-rank tests with Bonferroni correction for multiple comparisons. iSCl, incomplete spinal cord injury; VM, vastus medialis; TA, tibialis anterior; GM, gastrocnemius; ST, semitendinosus. 
A

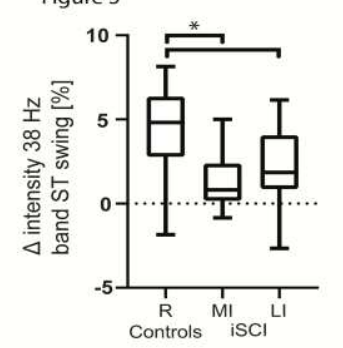

B

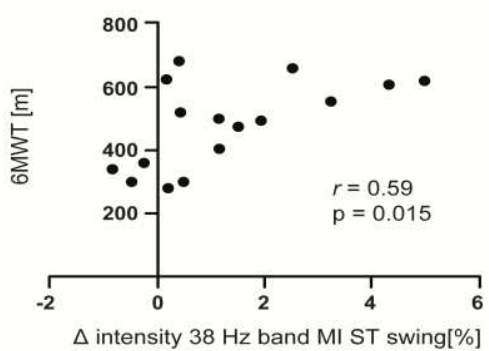

C

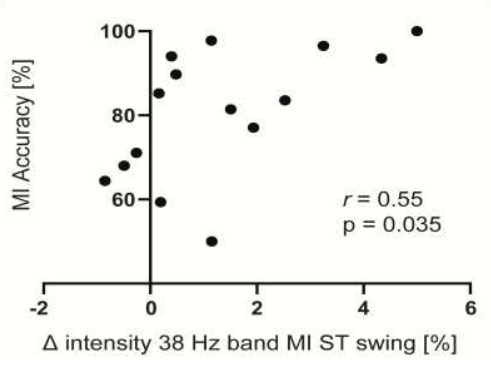

D
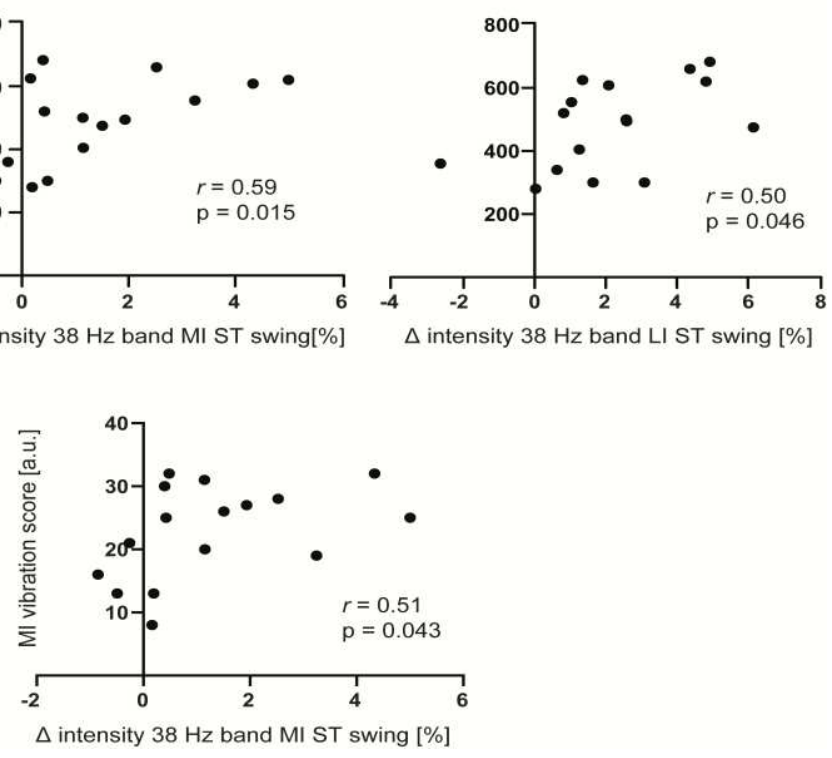

Figure 5: Comparison of the delta in the $38 \mathrm{~Hz}$ band (wavelet analysis) between controls and individuals with iSCl and between different impairment levels within the iSCl group.

A) Boxplot (whiskers: range) of the delta (targeted-normal walking) in the $38 \mathrm{~Hz}$ band in ST during swing for the control group $(n=21)$ and the iSCl group $(n=16)$. Asterisks indicate a significant difference between groups $(\alpha=0.05)$ tested by Post-Hoc comparisons of an ANOVA. B) Scatter plots of the performance in the $6 \mathrm{MWT}$ and the delta in the $38 \mathrm{~Hz}$ band of the $\mathrm{MI}$ and LI ST during swing for the iSCI group. Results of a Pearson correlation are indicated. C) Scatter plots of the accuracy during targeted walking and the delta in the 38 $\mathrm{Hz}$ band of the MI ST during swing for the iSCI group. Results of a Spearman correlation are indicated. D) Scatter plots of the clinical vibration score (test for proprioception) and the delta in the $38 \mathrm{~Hz}$ band of the MI and LI ST during swing for the iSCI group. Results of a Spearman correlation are indicated. iSCl, incomplete spinal cord injury; RMS, root mean square; R, right; MI, more impaired; LI, less impaired; ST, semitendinosus; 6MWT; 6minute walking test 


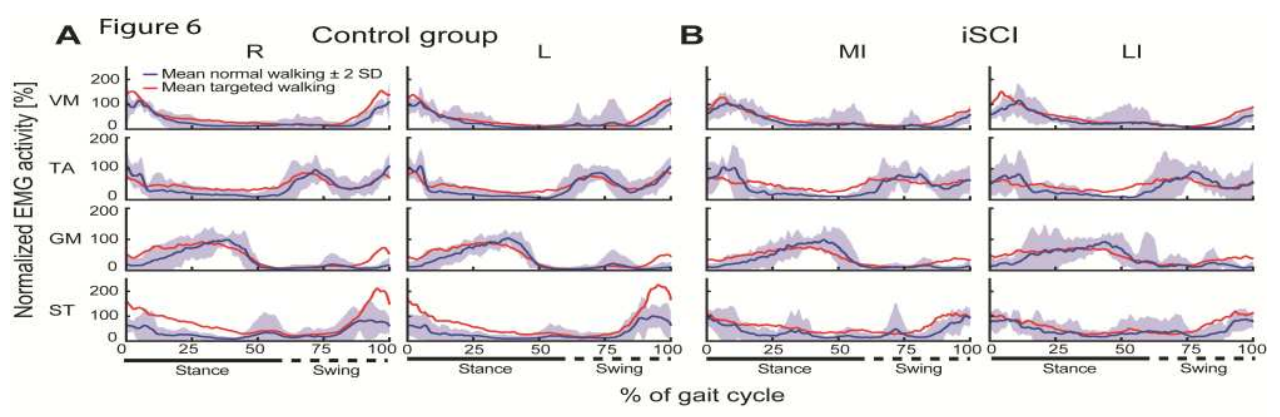

C

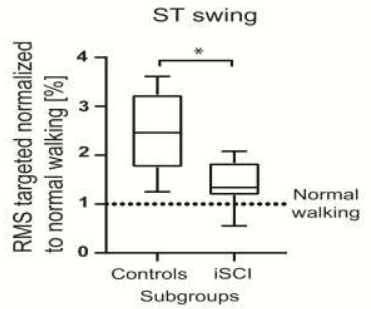

D

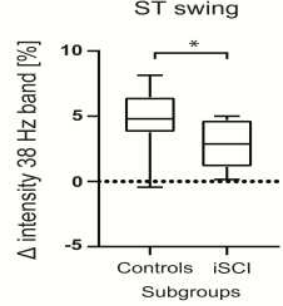

Figure 6: EMG characteristics during normal and targeted walking in two speed-matched subgroups of control participants and individuals with iSCI. A) Rectified and averaged EMG activity of the control subgroup during normal and targeted walking for right and left VM (r: $n=6 ; 1: n=6), T A(r: n=7 ; 1: n=7), G M(r: n=7 ; 1: n=7)$ and ST (r:n=7; I: $n=7)$. The EMG amplitudes were normalized to the $95^{\text {th }}$ percentile intensity during normal walking. B) Rectified and averaged EMG activity of the iSCI subgroup $(n=6)$ during normal and targeted walking for less and more impaired VM, TA, GM and ST. The EMG amplitudes were normalized to the $95^{\text {th }}$ percentile intensity during normal walking. C) Boxplots (whiskers: range) of RMS swing of ST (both leg) for subgroup of controls $(n=7)$ and individuals with iSCl ( $n=6)$. The asterisk indicates a significant difference between the groups ( $\alpha=0.05)$ tested by a Mann-Whitney-Test. D) Boxplots (whiskers: range) of delta between normal and targeted walking in ST during swing (both leg) for subgroup of controls ( $n=7$ ) and individuals with iSCI $(n=6)$. The asterisk indicates a significant difference between the groups ( $\alpha=0.05$ ) tested by a Mann-Whitney-Test. iSCl, incomplete spinal cord injury; RMS, root mean square; $R$, right; L, left; $\mathrm{MI}$, more impaired; LI, less impaired; SD, standard deviation; VM, vastus medialis; TA, tibialis anterior; GM, gastrocnemius; ST, semitendinosus. 
Supplementary table 1: Side differences of controls in EMG frequency characteristics during stance. Difference of relative intensity between normal and targeted walking in each frequency band of controls during physiological muscle activity phases in stance. Pvalues indicate the results of Mann-Whitney- $U$ tests to compare the two body sides and are Bonferroni corrected (for 9 frequency bands per muscle). VM, vastus medials; TA, tibialis anterior; GM, Gastrocnemius; ST, semitendinosus.

\begin{tabular}{|c|c|c|c|c|c|c|}
\hline & & VM & & & TA & \\
\hline & $\begin{array}{c}\text { Difference } \\
\text { right [\%] }\end{array}$ & $\begin{array}{c}\text { Difference } \\
\text { left [\%] }\end{array}$ & P-value & $\begin{array}{c}\text { Difference } \\
\text { right [\%] }\end{array}$ & $\begin{array}{c}\text { Difference } \\
\text { left [\%] }\end{array}$ & P-value \\
\hline $7 \mathrm{~Hz}$ & $+1.4 \pm 2.4$ & $+1.0 \pm 1.4$ & 1.00 & $-0.2 \pm 2.0$ & $-0.2 \pm 0.8$ & 1.00 \\
\hline $19 \mathrm{~Hz}$ & $+1.0 \pm 2.3$ & $+1.2 \pm 1.6$ & 1.00 & $+0.3 \pm 1.6$ & $0.0 \pm 1.5$ & 1.00 \\
\hline $38 \mathrm{~Hz}$ & $+0.4 \pm 1.3$ & $+0.6 \pm 1.3$ & 1.00 & $+0.8 \pm 1.1$ & $+0.6 \pm 1.3$ & 1.00 \\
\hline $62 \mathrm{~Hz}$ & $-0.3 \pm 1.2$ & $-0.8 \pm 1.3$ & 1.00 & $-0.1 \pm 0.9$ & $+0.3 \pm 0.9$ & 0.83 \\
\hline $92 \mathrm{~Hz}$ & $-0.8 \pm 1.6$ & $-1.0 \pm 1.3$ & 1.00 & $-0.3 \pm 0.9$ & $-0.2 \pm 1.0$ & 1.00 \\
\hline $128 \mathrm{~Hz}$ & $-0.6 \pm 1.4$ & $-0.1 \pm 0.8$ & 1.00 & $0.0 \pm 0.8$ & $-0.1 \pm 0.9$ & 1.00 \\
\hline $170 \mathrm{~Hz}$ & $-0.5 \pm 0.7$ & $-0.2 \pm 0.9$ & 1.00 & $0.0 \pm 0.8$ & $+0.1 \pm 0.8$ & 1.00 \\
\hline $218 \mathrm{~Hz}$ & $-0.3 \pm 0.5$ & $-0.3 \pm 0.6$ & 1.00 & $-0.2 \pm 0.7$ & $-0.2 \pm 0.8$ & 1.00 \\
\hline \multirow[t]{2}{*}{$271 \mathrm{~Hz}$} & $-0.3 \pm 0.5$ & $-0.4 \pm 0.8$ & 1.00 & $-0.2 \pm 0.6$ & $-0.2 \pm 0.5$ & 1.00 \\
\hline & & GM & & & ST & \\
\hline $7 \mathrm{~Hz}$ & $+0.6 \pm 0.6$ & $+0.8 \pm 0.4$ & 1.00 & $+1.2 \pm 3.0$ & $+1.3 \pm 2.6$ & 1.00 \\
\hline $19 \mathrm{~Hz}$ & $+0.9 \pm 1.1$ & $+1.4 \pm 1.1$ & 1.00 & $+0.8 \pm 3.4$ & $+0.4 \pm 2.9$ & 1.00 \\
\hline $38 \mathrm{~Hz}$ & $+0.8 \pm 1.0$ & $+0.7 \pm 1.2$ & 1.00 & $+0.5 \pm 1.6$ & $+1.1 \pm 2.9$ & 1.00 \\
\hline
\end{tabular}




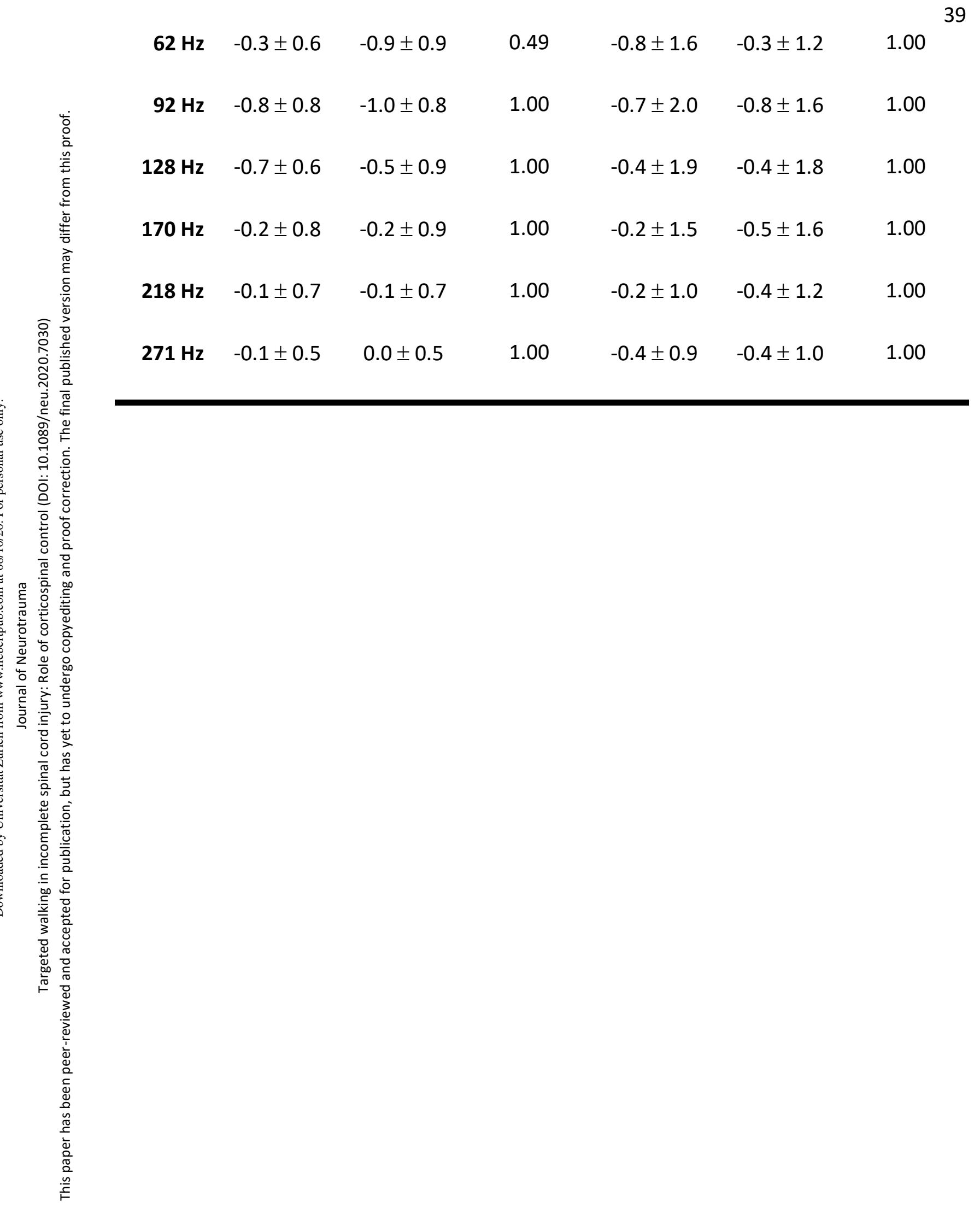


Supplementary table 2: Side differences of controls in EMG frequency characteristics during swing. Difference of relative intensity between normal and targeted walking in each frequency band of controls during physiological muscle activity phases in swing. Pvalues indicate the results of Mann-Whitney- $U$ tests to compare the two body sides and are Bonferroni corrected (for 9 frequency bands per muscle). VM, vastus medials; TA, tibialis anterior; GM, Gastrocnemius; ST, semitendinosus.

\begin{tabular}{|c|c|c|c|c|c|c|}
\hline & & VM & & & TA 1 & \\
\hline & $\begin{array}{c}\text { Difference } \\
\text { right [\%] }\end{array}$ & $\begin{array}{c}\text { Difference } \\
\text { left [\%] }\end{array}$ & P-value & $\begin{array}{c}\text { Difference } \\
\text { right [\%] }\end{array}$ & $\begin{array}{c}\text { Difference } \\
\text { left [\%] }\end{array}$ & P-value \\
\hline $7 \mathrm{~Hz}$ & $+0.2 \pm 0.9$ & $-0.1 \pm 1.3$ & 1.00 & $+0.5 \pm 1.1$ & $+0.4 \pm 0.4$ & 1.00 \\
\hline $19 \mathrm{~Hz}$ & $+0.8 \pm 1.4$ & $+0.6 \pm 1.5$ & 1.00 & $0.0 \pm 1.3$ & $+0.4 \pm 1.2$ & 1.00 \\
\hline $38 \mathrm{~Hz}$ & $+0.9 \pm 1.6$ & $+1.0 \pm 1.5$ & 1.00 & $0.0 \pm 1.2$ & $0.0 \pm 0.8$ & 1.00 \\
\hline $62 \mathrm{~Hz}$ & $-0.5 \pm 1.5$ & $+0.1 \pm 1.3$ & 1.00 & $-0.2 \pm 0.9$ & $-0.3 \pm 0.9$ & 1.00 \\
\hline $92 \mathrm{~Hz}$ & $-0.3 \pm 1.5$ & $-0.3 \pm 1.6$ & 1.00 & $-0.3 \pm 0.7$ & $-0.3 \pm 1.0$ & 1.00 \\
\hline $128 \mathrm{~Hz}$ & $-0.1 \pm 0.8$ & $0.0 \pm 1.2$ & 1.00 & $0.0 \pm 0.8$ & $-0.2 \pm 0.9$ & 1.00 \\
\hline $170 \mathrm{~Hz}$ & $-0.1 \pm 0.8$ & $0.0 \pm 0.9$ & 1.00 & $0.0 \pm 0.5$ & $-0.0 \pm 0.7$ & 1.00 \\
\hline $218 \mathrm{~Hz}$ & $-0.4 \pm 0.7$ & $-0.4 \pm 0.8$ & 1.00 & $-0.1 \pm 0.5$ & $+0.1 \pm 0.4$ & 1.00 \\
\hline \multirow[t]{2}{*}{$271 \mathrm{~Hz}$} & $-0.5 \pm 0.6$ & $-0.5 \pm 1.0$ & 1.00 & $+0.1 \pm 0.3$ & $0.0 \pm 0.4$ & 1.00 \\
\hline & & TA 2 & & & ST & \\
\hline $7 \mathrm{~Hz}$ & $+0.7 \pm 1.9$ & $+0.4 \pm 1.0$ & 1.00 & $+0.9 \pm 1.1$ & $+0.6 \pm 2.0$ & 1.00 \\
\hline $19 \mathrm{~Hz}$ & $+1.5 \pm 1.9$ & $+1.4 \pm 1.8$ & 1.00 & $+3.3 \pm 2.2$ & $+2.9 \pm 2.1$ & 1.00 \\
\hline $38 \mathrm{~Hz}$ & $+0.9 \pm 1.9$ & $+1.2 \pm 1.4$ & 1.00 & $+4.5 \pm 2.7$ & $+5.2 \pm 3.0$ & 1.00 \\
\hline
\end{tabular}




$\begin{array}{rrrrrrr}\mathbf{6 2 ~ H z} & -0.2 \pm 1.1 & +0.1 \pm 0.8 & 1.00 & +1.0 \pm 1.7 & +1.5 \pm 1.5 & 1.00 \\ \mathbf{9 2 ~ H z} & -0.9 \pm 1.3 & -0.7 \pm 1.3 & 1.00 & -2.3 \pm 1.8 & -2.0 \pm 1.2 & 1.00 \\ 128 \mathrm{~Hz} & -0.6 \pm 1.5 & -0.9 \pm 1.3 & 1.00 & -2.4 \pm 1.5 & -2.8 \pm 1.8 & 1.00 \\ \mathbf{1 7 0 ~ H z} & -0.4 \pm 0.6 & -0.5 \pm 1.1 & 1.00 & -1.7 \pm 1.6 & -2.1 \pm 1.8 & 1.00 \\ 218 \mathrm{~Hz} & -0.5 \pm 0.6 & -0.5 \pm 1.2 & 1.00 & -1.7 \pm 1.0 & -1.7 \pm 1.3 & 1.00 \\ & & & & & & \\ \mathbf{2 7 1 ~ H z} & -0.4 \pm 0.7 & -0.6 \pm 0.7 & 1.00 & -1.6 \pm 0.9 & -1.6 \pm 1.1 & 1.00\end{array}$


Supplementary table 3: Subgroup characteristics. Characteristics of the two speedmatched subgroups (mean \pm SD or median \pm range for clinical score). P-values for the statistical comparisons between the groups (independent t-test for age, height weight, 6MWT, speed, maximal step length \& width, precision; Chi-square test for gender MannWhitney U-test for motor score, sensory scores (light touch \& pinprick), vibration, position sense and modified Ashworth scale). 6MWT; 6-minute walking test; n/a, not applicable.

Control group

iSCl

p-value

n

7

6

$\mathrm{n} / \mathrm{a}$

Gender [female : male]

$3: 4$

$2: 4$

0.72

Age [years]

$62.4 \pm 8.6$

$52.5 \pm 11.3$

0.09

Height [cm]

$170.0 \pm 7.7$

$173.2 \pm 6.3$

0.44

Weight [kg] $\quad 75.2 \pm 12.5$

$76.8 \pm 18.5$

0.87

6MWT [m] $\quad 650 \pm 84.1$

$625 \pm 44$

0.52

$50 \%$ of maximal speed

$3.4 \pm 0.3$

$3.4 \pm 0.2$

0.57

[km/h]

Maximal step length [cm]

$141.6 \pm 8.1$

$158 \pm 18.2$

0.05

Maximal step width [cm]

$30.0 \pm 2.9$

$30.2 \pm 4.2$

0.93

Precision [\%]

$95.2 \pm 5.7$

$93.9 \pm 6.1$

0.87

AIS

$\mathrm{n} / \mathrm{a}$

$D(6)$

$\mathrm{n} / \mathrm{a}$

cervical (3)

Lesion level

$\mathrm{n} / \mathrm{a}$

thoracic (3)

traumatic (3)

Cause

$\mathrm{n} / \mathrm{a}$

n/a

ischemic (2) 
disc herniation (1)

Lower extremity motor

Sensory score light touch

(max. 112)

Sensory score pin-prick

(max. 112)

Vibration sense lower limbs

(max. 64)

Position sense lower limb

(max. 4)

Modified Ashworth scale

(max. 16)
$50 \pm 0$

$112 \pm 0$

$112 \pm 0$

$60 \pm 8$

$4 \pm 0$

$4 \pm 3$

$0 \pm 2.5$

$0 \pm 0$

$55 \pm 34$

0.12

0.02

$106.5 \pm 29$

0.46

0.46 